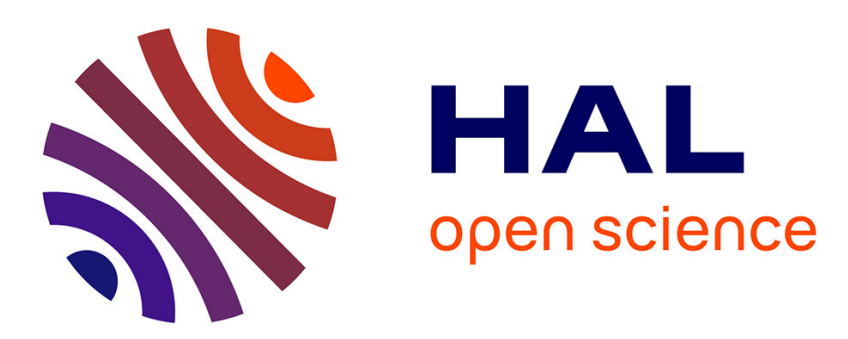

\title{
Skills and Limitations of the Adiabatic Omega Equation: How Effective Is It to Retrieve Oceanic Vertical Circulation at Mesoscale and Submesoscale?
}

\author{
Alice Pietri, Xavier Capet, Francesco d'Ovidio, Marina Lévy, Julien Le \\ Sommer, Jean-Marc Molines, Hervé Giordani
}

\section{To cite this version:}

Alice Pietri, Xavier Capet, Francesco d'Ovidio, Marina Lévy, Julien Le Sommer, et al.. Skills and Limitations of the Adiabatic Omega Equation: How Effective Is It to Retrieve Oceanic Vertical Circulation at Mesoscale and Submesoscale?. Journal of Physical Oceanography, 2021, 51 (3), pp.931-954. 10.1175/jpo-d-20-0052.1 . hal-03096579

\section{HAL Id: hal-03096579 \\ https://hal.science/hal-03096579}

Submitted on 5 Jan 2021

HAL is a multi-disciplinary open access archive for the deposit and dissemination of scientific research documents, whether they are published or not. The documents may come from teaching and research institutions in France or abroad, or from public or private research centers.
L'archive ouverte pluridisciplinaire HAL, est destinée au dépôt et à la diffusion de documents scientifiques de niveau recherche, publiés ou non, émanant des établissements d'enseignement et de recherche français ou étrangers, des laboratoires publics ou privés. 
1. Skills and limitations of the adiabatic omega equation: how effective is it to retrieve oceanic vertical circulation at meso and submesoscale?

\author{
Alice Pietri ${ }^{* \dagger}$, Xavier Capet, Francesco d'Ovidio, Marina Levy \\ Laboratoire dOcéanographie et du Climat, Institut Pierre Simon Laplace (LOCEAN, \\ CNES/CNRS/IRD/MNHN/SU), Paris, France \\ Julien Le Sommer, Jean-Marc Molines \\ Univ. Grenoble-Alpes (CNRS/IRD/IGE), Grenoble, France \\ Hervé Giordani \\ Centre National de Recherches, Météorologiques, Météo-France, Toulouse, France
}

${ }_{10}^{*}$ Corresponding author address: Alice Pietri, LOCEAN-IPSL/CNES, Jussieu, Paris.

11 E-mail: alice.pietri@ locean-ipsl.upmc.fr

${ }_{12}^{\dagger}$ Current affiliation: Instituto del Mar del Peru (IMARPE), Callao, Perú. 
ABSTRACT 
${ }_{14}$ widely used methods to reconstruct vertical velocity $(w)$ from in-situ data.

${ }_{15}$ As observational networks with much higher spatial and temporal resolutions

${ }_{16}$ are being designed, the question rises of identifying the approximations and

${ }_{17}$ scales at which an accurate estimation of $w$ through the omega equation can

${ }_{18}$ be achieved and what are the critical scales and observables needed. In this

${ }_{19}$ paper we test different adiabatic omega reconstructions of $w$ over several re-

${ }_{20}$ gions representative of main oceanic regimes of the global ocean in a fully

${ }_{21}$ eddy-resolving numerical simulation with a $1 / 60^{\circ}$ horizontal resolution. We

${ }_{22}$ find that the best reconstructions are observed in conditions characterized by

23 energetic turbulence and/or weak stratification where near-surface frontal pro-

${ }_{24}$ cesses are felt deep into the ocean interior. The quasi-geostrophic omega

equation gives satisfactory results for scales larger than $\sim 10 \mathrm{~km}$ horizon-

tally while the improvements using a generalized formulation are substantial only in conditions where frontal turbulent processes are important (providing improvements with satisfactory reconstruction skill down to $\sim 5 \mathrm{~km}$ in scale). The main sources of uncertainties that could be identified are related to processes responsible for ocean thermal wind imbalance (TWI), which is particularly difficult to account for (especially in observation-based studies) and to the deep flow which is generally improperly accounted for in omega reconstructions through the bottom boundary condition. Nevertheless, the reconstruction of mesoscale vertical velocities may be sufficient to estimate vertical fluxes of oceanic properties in many cases of practical interest. 


\section{Introduction}

In geophysical fluids, the combined effect of stratification and rotation strongly inhibits vertical velocities over a broad range of horizontal scales $\left(\mathrm{L}_{h}\right)$, near and above the so-called deformation radius ( $\mathrm{Rd})$, typically $30 \mathrm{~km}$ in the ocean. Vertical velocities remain small even for $\mathrm{L}_{h}$ much below Rd (Pollard and Regier 1992; Giordani et al. 2006), typically a few meters to several tens of meters per day for submesoscale motions, $\mathrm{L}_{h} \sim \mathrm{O}(1) \mathrm{km}$, although their intensity tends to increase somewhat at finer scales. Much weaker than horizontal advection, vertical transport of oceanic properties such as heat and biogeochemical tracers is nevertheless of crucial importance for the overall functioning of the world ocean (Lévy et al. 2012a). In some regions, atmospheric forcings are such that the large-scale flow (100 km and larger) has a vertical component, e.g., under the influence of coastal upwelling favourable winds or positive wind stress curl. At finer scale $(1-100 \mathrm{~km})$ intermittent vertical velocities are associated with the mesoscale and submesoscale turbulence and can be generated by forced and unforced motions such as frontogenesis, baroclinic instabilities or air-sea interactions, with possible coupling between them (e.g. Thomas and Lee 2005). They are responsible for vertical fluxes that have proved difficult to quantify but are widely believed to play a major role in the heat (Ferrari 2011; Su et al. 2020; Siegelman et al. 2020) and salt budgets (Lien et al. 2014), in the carbon and nutrient cycles (Ledwell et al. 2008; Balwada et al. 2018), and in shaping oceanic biodiversity (Lévy et al. 2010, 2012b, 2014, 2018; Siegelman et al. 2020). It should be noted that vertical velocities also vary at the scales smaller than the submesoscale in particular vertical fluxes associated with 3D turbulence (Whitt et al. 2019). However the submesoscale-mesoscale (hereafter SMS) range on which this study focuses is important because i) it presumably contains a large fraction of co-variance between $w$ and many key tracer fields (Ledwell et al. 2008; Chenillat et al. 2015; Balwada et al. 2018), ii) SMS vertical motions 
can be coherent over relatively long/large time/space scales (including vertically) such that they produce long range vertical displacements and fluxes, e.g., , organic and inorganic carbon from the euphotic layer into the dark ocean (Boyd et al. 2019).

Direct measurements of vertical velocities with SMS spatiotemporal resolution would thus be highly desirable. However the magnitude of $w$ at these scales is typically $\mathrm{O}(1) \mathrm{mm} \mathrm{s}^{-1}(\sim 100 \mathrm{~m}$ day $^{-1}$ ) or less (e.g., up to $2-3 \mathrm{~mm} \mathrm{~s}^{-1}$ in the highly turbulent Gulf Stream, Lindstrom and Watts 1994) which places them below the noise level of any existing current meter for the typical time scales of a few days to weeks over which they vary. Different ingenious ways to circumvent this difficulty have been developed over time. For instance, direct integrated measurements of vertical displacements $\int w d t$ have been made using Lagrangian drifters (Bower and Rossby 1989; D’Asaro et al. 2004; Steffen and D'Asaro 2002). Alternatively, indirect reconstruction methods have been proposed based on the heat/density conservation equation (Strass 1994; Lindstrom et al. 1997; Yu et al. 2019), the vorticity equation (Strass 1994; Giordani et al. 2005) or the 3D non-divergence of the flow (Helber and Weisberg 2001; Horii et al. 2011). A different framework has also emerged to infer vertical velocities from the theory of surface quasigeostrophy (SQG) (Held et al. 1995; Lapeyre and Klein 2006a; LaCasce and Mahadevan 2006), where the 3D flow structure can be essentially determined from the knowledge of the surface buoyancy field (Isern-Fontanet et al. 2006; Klein et al. 2009; Ponte and Klein 2013; Qiu et al. 2020). Overall, the most commonly used method to infer $w$ is based on frontogenetic theories and the so-called omega equation, which is the subject of the present study.

Quasi-geostrophic and semi-geostrophic versions of the omega equation (Hoskins et al. 1978) have been applied for decades (Leach 1987; Pollard and Regier 1992). The former and to a lesser extent the latter are by nature suited to low Rossby number environments, i.e., a priori away from regions of intense vertical velocities. A generalized version of the omega equation was first intro- 
duced in the atmospheric community (Davies-Jones 1991; Pauley and Nieman 1992; Giordani and Caniaux 2001) and subsequently applied to the ocean (Viúdez et al. 2002; Giordani et al. 2006). Vertical velocity forcing processes present in this generalized omega equation are kinetic deformation that arises in shear and confluence situations, mixing and momentum diffusion which can also disrupt the thermal wind balance and an additional prognostic term due to the rate of change of unbalanced motions. This latter term shall be zero under the quasi-geostrophic approximation and is systematically neglected in ocean applications, which makes the omega equation diagnostic for $w$ (see Qiu et al. 2020 for the only evaluation of this term that we know of). Despite this simplification, the generalized formulations of the omega equation are expected to hold even in regions where the flow exhibits high Rossby number (Viúdez et al. 2002; Viúdez and Dritschel 2004; Shearman et al. 2000). Since the omega equation is most frequently used to infer $w$ from observations the chosen formulation usually depends on the data available. The adiabatic quasigeostrophic version of the equation only requires density observations and a reference level to derive geostrophic currents from the thermal wind balance. Knowledge of the absolute horizontal velocity field allows to take into account the effect of the ageostrophic deformation and advection. Solving more elaborate forms of the omega equation require additional data, such as atmospheric forcing (Giordani et al. 2006). Statistical methods based on multivariate empirical orthogonal functions can be used to determine the SMS ocean state from surface satellite information and sparse in situ data (e.g. ARMOR3D high resolution operational product, Guinehut et al. 2012). Those methods have been used regularly to infer the forcing terms for omega inversions (Buongiorno Nardelli and Santoleri 2005; Buongiorno Nardelli et al. 2012; Buongiorno Nardelli et al. 2018; Barceló-Llull et al. 2018; Buongiorno Nardelli 2020).

Numerous investigations of oceanic vertical velocities based on the omega equation have been carried out since the 1980s, most frequently in the context of mesoscale-resolving observational 
efforts. A few of them have used independent ways to estimate $w$ and evaluate the skills of the omega reconstructions. Conclusions are generally that the omega equation has reasonable skills at the mesoscale. A few studies have also applied this equation to numerical model outputs to test its reliability and degree of accuracy (Pinot et al. 1996; Allen et al. 2001; Rixen et al. 2003; Uchida et al. 2019). The domain of validity and typical errors attached to an omega reconstruction for realistic cases are however not clearly established. This is particularly true at submesoscale where the omega equation has been increasingly applied (Pallàs-Sanz et al. 2010). The benefit that one can expect from using more elaborate versions of the omega equation compared to the quasigeostrophic formulation are also not clearly established. An overview of the literature on the subject offers a great diversity of conclusions (from major to no benefits or even degradation of the reconstruction). We provide a review of omega-reconstructions literature in the the bibliography table (SI1). Overall, this literature appears to be mainly composed of an accumulation of test cases (Pascual et al. 2017; Buongiorno Nardelli et al. 2012; Rixen et al. 2003; Allen et al. 2001), many of which are in idealized settings (Viúdez and Dritschel 2004). These individual cases can be difficult to compare against one another.

The main novelty of the present study is to apply the same analysis framework to assess the skills of the adiabatic omega equation in several dynamical regimes representative of a broad diversity of ocean conditions. The aim is to propose a more integrated and comprehensive understanding of the skills and limitations of omega reconstructions. Given this ambition, we restrict the scope somewhat by mainly focusing on vertical velocities outside mixed layers, typically at depths $\sim$ $200-400 \mathrm{~m}$. Submesoscale velocities in the mixed layer are important too (e.g., Smith et al. 2016) but they more strongly compete and interact with 3D turbulent processes responsible with intense mixing in ways that are just beginning to be clarified (Thomas and Lee 2005; Hamlington et al. 2014; Suzuki et al. 2016; Sullivan and McWilliams 2018; Callies and Ferrari 2018). On 
the other hand, we expect SMS vertical velocities obtained by means of omega reconstruction to be more readily useful to estimate tracer fluxes below the mixed layer, typically upward fluxes of nutrient into the euphotic layer (Pascual et al. 2015) or downward fluxes of oxygen into subsurface hypoxic layers (Thomsen et al. 2016).

Exploring the ability of the adiabatic omega equation to reconstruct $w$ in our realistic, eddyresolving, circulation model reveals that, in this depth range, reconstruction skills turn out to be strongly sensitive to the dynamical regime under consideration, but also to the physical (length) scale of interest. In particular, it is shown that omega reconstructions perform well for scales down to $\sim 10 \mathrm{~km}$ while rapid degradation occurs at smaller scales. Consequently, the best results are obtained in conditions characterized by high vertical velocity variance which are found in regions with intense mesoscale frontogenesis. On the other hand, relaxing the QG assumptions with the generalized formulation is shown to have a modest impact on the reconstruction. Instead, an analysis of the relative importance of the sources of errors reveals that a major impact can be attributed to the choice of the bottom boundary conditions (BBC). The impact of neglecting vertical mixing terms, though presently deemed limited to the upper layers, would also require dedicated investigations.

The paper is organized as follows. Data and methods are presented in section 2. In section 4 vertical velocity reconstructions in the different dynamical regimes retained for this study are evaluated. A series of sensitivities allows us to test the impact of the level of complexity of the omega equation; the formulation of the discrete problem; and several possible choices related to the boundary conditions. Some reflections on the sources of errors and the dynamical environment are offered in the discussion. 


\section{Data and Methods}

Mathematical symbols have their usual meaning. $T, S, \rho$ and $\sigma_{t}$ refer to (respectively) potential temperature, salinity, density and potential density anomaly. $x$ (resp. $y$ and $z$ ) and $u$ (resp. $v$ and w) refer to zonal (resp. meridional and vertical) directions and velocity. More precisely in the case of vertical velocities we will distinguish true velocities $w$ from reconstructed velocities denoted $\omega$ with a subscript that refers to the precise omega equation formulation that is being used (see below Sec. $2 b) . N^{2}$ is the buoyancy frequency.

\section{a. The NATL model}

The outputs from a submesoscale-permitting numerical simulation are used to reconstruct vertical velocity fields using different versions of the omega equation and compare them to the ground truth model vertical velocities $w_{\text {model }}$. The NATL60 MJM155 simulation is run using the NEMO v3.5 code. It has a horizontal resolution of $1 / 60^{\circ}(\mathrm{dx}$ comprised between 0.8 and $1.6 \mathrm{~km}$ depending on the latitude), yielding an effective resolution of $\sim 10 \mathrm{dx}(\sim 10-15 \mathrm{~km})$ (Soufflet et al. 2016). This means that a turbulent feature associated with a typical wavelength around $\sim 10-15$ $\mathrm{km}$ (typical scale $\sim 1.5-2 \mathrm{~km}$ ) are energetically subjected to a negligible influence of the dissipation operator. Below those scales numerical errors can arise that will affect the representation of physical processes although the misrepresented dynamics in the model solutions may still share important properties with the dynamics of the system obtained after resolution convergence (Le Sommer et al. 2018). On the vertical, the simulation has 300 levels (dz increases with depth and ranges from 1 to $30 \mathrm{~m}$ ) and in the range of depths of analysis (200-400 $\mathrm{m}$ depth), the vertical resolution is $\sim 10 \mathrm{~m}$. The simulation is forced by realistic atmospheric forcings (DFS5.2) and boundary conditions (GLORYS2V3). It was integrated over a 5 years period (2004-2008) (Amores et al. 2018; Ducousso et al. 2017; Fresnay et al. 2018). 
The model domain encompasses the whole North Atlantic. For this study, four contrasted sectors were selected. For each region, eleven consecutive daily averaged outputs are analysed for two opposite seasons of the year, in June and December. Near-inertial motions produced by the highfrequency variability of the atmospheric forcings are partly filtered out and partly aliased in model $u, v, \sigma_{t}$ and $w$ daily fields that are used in this study. A cleaner separation between balanced and unbalanced motions would be useful to untangle their respective roles (e.g., see Qiu et al. 2020). Lacking the high-frequency model outputs needed to do better, we note that, in most situations, omega estimations made using observations contain an unknown but presumably larger inertia-gravity (IG) wave contribution. Our study can thus be considered as a favorable evaluation in which this source of errors is minimized by the daily averaging. Absence of tidal forcing in NATL60 will also tend to underestimate the energy level of unbalanced motion (Qiu et al. 2018) and thus has a similar consequence. The relevance of the study to the real ocean context is however justified by the fact that model vertical velocities in the submesoscale-permitting class of simulations are known to capture the patterns expected from the theory and observation based studies.

The four regions we focus on are:

- the Gulf Stream/LatMix region (Shcherbina et al. 2013, LMX) centered around $38^{\circ} \mathrm{N}$ and $67^{\circ} \mathrm{W}$. It encompasses the very energetic Gulf Stream current. It is characterized by intense mesoscale activity composed of meanders and eddies. Vertical motions in the Gulf Stream region have been the subject of many studies aimed at describing water exchanges between the jet core and its vicinities (Bower and Rossby 1989; Lindstrom and Watts 1994; Joyce et al. 2013). This environment and the large amplitude meanders of the Gulf Stream in particular are well known to produce intense vertical velocities (Fig. 1a). 
- The region of the Azores current (AZO) centered at $33^{\circ} \mathrm{N}, 34^{\circ} \mathrm{W}$ which is characteristic of a subtropical regime with limited atmospheric forcing and modest mesoscale activity. It is the least energetic of the four regions in terms of vertical velocities (Fig. 1b). We relate this to the modest role played by surface density contrasts and upper ocean frontogenesis in the regional dynamics (Lapeyre 2009; Volkov and Fu 2010, 2011)

- An area of the subpolar gyre South West of Iceland encompassing part of the Rekjanes ridge (REK) and centered around $54^{\circ} \mathrm{N}, 31^{\circ} \mathrm{W}$. The regional dynamics is characterized by weak mean flow and a moderately intense mesoscale activity made of deep reaching isolated structures (Fig. 1c).

- A sector of the Atlantic North-Eastern margin near $49^{\circ} \mathrm{N}$ and $15^{\circ} \mathrm{W}$, where the Osmosis experiment took place (Buckingham et al. 2016, OSM). The regional dynamics is characterized by weak large scale circulation, weak mesoscale activity, and a marked seasonality of the submesoscale activity which is very intense in the wintertime (Fig. 1d; Thompson et al. 2016).

To limit the computational cost of inverting the omega equation a 3D sub-domain of each region is retained with dimensions $\sim 360$ by $\sim 270 \mathrm{~km}$ on the horizontal and $1600 \mathrm{~m}$ in the vertical (reduced depth ranges will also be used in sensitivity tests section 4d). Horizontal (native) resolution is $\sim 1.5 \mathrm{~km}$ while the data is linearly interpolated on a regular vertical grid with a $5 \mathrm{~m}$ resolution. For each region, a particular depth is selected to perform some of our analyses. As presented in the introduction the spirit of this investigation led us to choose depth levels $50 \mathrm{~m}$ below the deepest winter mixed layers encountered in that region so near-surface frontal processes are attenuated at this depth and our study is useful to make progress on biogeochemical fluxes between the mixed layer and the ocean interior. Specifically, selected depths of analysis (referred to as $z_{a}$ ) are $220 \mathrm{~m}$ for LMX, $250 \mathrm{~m}$ for AZO, and $380 \mathrm{~m}$ for REK and OSM. 


\section{b. Formulations of the omega equation}

The underlying principles of the omega equation combine two lines of argument (Hoskins 1982)

- kinematical: a turbulent flow stirring an heterogeneous surface buoyancy field produces regions of gradient intensification. Specifically, frontal intensification is promoted in the confluence and shear situations that are frequently encountered in mesoscale turbulent conditions (e.g. Fig. 2).

- dynamical: the thermal wind balance, which should approximately hold at meso/submesoscale, is disrupted in situations of frontal intensification where density gradients are enhanced while the vertical velocity shear is being reduced. This leads to the development of ageostrophic secondary circulations (ASC) that attempt to thwart thermal imbalance by restoring shear and slumping isopycns. In the ocean interior this process is very efficient at preventing frontal intensification which is a further justification for the weakness of vertical velocities away from the surface. At the air-sea interface, the upper boundary condition $w \approx 0$ limits the efficiency of ageostrophic circulations so frontal intensification can generally proceed further and yield intense vertical velocities.

Mathematically, the frontogenesis process is captured by a relationship between the spatial derivatives of $w$ and a forcing term expressed as the divergence of a vector of forcings $\mathbf{Q}$

$$
\mathscr{L}(w)=\nabla \cdot \mathbf{Q}
$$

$\mathscr{L}$ is a second-order differential operator that can also take different forms depending on which processes responsible for thermal wind disruptions are being considered in $\mathbf{Q}$. 

ing hydrostatic and Boussinesq flows:

$$
\begin{aligned}
& \frac{d u}{d t}-f v=-\frac{1}{\rho_{0}} \frac{\partial p}{\partial x}+F_{x} \\
& \frac{d v}{d t}+f u=-\frac{1}{\rho_{0}} \frac{\partial p}{\partial y}+F_{y} \\
& \frac{d \rho}{d t}=F_{\rho} \\
& \nabla \cdot \mathbf{v}=0
\end{aligned}
$$

where $\left(F_{x}, F_{y}\right)$ and $F_{\rho}$ are source/sink of momentum and buoyancy caused by turbulent mixing.

The flow is then decomposed into a geostrophic $\left(\mathbf{v}_{\mathbf{g}}\right)$ and ageostrophic $\left(\mathbf{v}_{\mathbf{a g}}\right)$ component

$$
\mathbf{v}=\mathbf{v}_{\mathbf{g}}+\mathbf{v}_{\mathbf{a g}}
$$

where the geostrophic velocity satisfies the thermal wind balance:

$$
\left\{\begin{array}{l}
f \frac{\partial u_{g}}{\partial z}=\frac{g}{\rho} \frac{\partial \rho}{\partial y} \\
f \frac{\partial v_{g}}{\partial z}=-\frac{g}{\rho} \frac{\partial \rho}{\partial x}
\end{array}\right.
$$

and the residual ageostrophic flow component departing from this balance (the so-called thermal wind imbalance - TWI) satisfies:

$$
\left\{\begin{array}{l}
f \frac{\partial u_{a g}}{\partial z}=f \frac{\partial u}{\partial z}-\frac{g}{\rho} \frac{\partial \rho}{\partial y} \\
f \frac{\partial v_{a g}}{\partial z}=f \frac{\partial v}{\partial z}+\frac{g}{\rho} \frac{\partial \rho}{\partial x}
\end{array}\right.
$$

The generalized omega equation (1) is obtained by manipulating the time evolution equation for the TWI and yields (Giordani and Planton 2000):

$$
f^{2} \frac{\partial^{2} w}{\partial z^{2}}+\nabla_{h}\left(N^{2} \cdot \nabla_{h} w\right)=\nabla \cdot \mathbf{Q}
$$


where the $\mathbf{Q}$ vector involved in the right hand side (rhs) of the equation can be expressed as a sum of different forcings:

$$
\mathbf{Q}=2 \underbrace{\left(\mathbf{Q}_{\mathrm{twg}_{\mathrm{g}}}+\mathbf{Q}_{\mathrm{tw}_{\mathrm{ag}}}\right)}_{\mathbf{Q}_{\mathrm{tw}}}+\mathbf{Q}_{\mathbf{d a g}}+\mathbf{Q}_{\mathrm{dr}}+\mathbf{Q}_{\mathrm{th}}+\mathbf{Q}_{\mathbf{d m}}
$$

following the notations of Giordani et al. (2006). $\mathbf{Q}_{\mathrm{tw}}$ is the kinematic deformation and can be decomposed into a geostrophic $\mathbf{Q}_{\mathbf{t w g}}$ and an ageostrophic component $\mathbf{Q}_{\mathbf{t w}_{\mathrm{ag}}} \cdot \mathbf{Q}_{\mathbf{d a g}}$ is the deformation of the thermal wind imbalance and $\mathbf{Q}_{\mathbf{d r}}$ its material rate of change. In all practical situations, $\mathbf{Q}_{\mathbf{d r}}$ cannot be estimated and is therefore unaccounted for in the remainder of the study. $\mathbf{Q}_{\mathbf{t h}}$ and $\mathbf{Q}_{\mathbf{d m}}$ refer respectively to the diabatic turbulent buoyancy and momentum forcings.

Being interested in the ability to determine vertical velocities through and below the thermocline we neglect the effects of diffusive momentum and buoyancy fluxes which are mainly active in or immediately below the mixed layer (Giordani et al. 2006; Yoshikawa et al. 2012; Thomas et al. 2010) but have a limited effect on subsurface velocities (Nagai et al. 2006; Yoshikawa et al. 2012). In fact, Xie et al. (2017), using microstructure shear measurements to infer the vertical diffusivity, derived the vertical mixing terms $\left(\mathbf{Q}_{\mathbf{t h}}\right.$ and $\left.\mathbf{Q}_{\mathbf{d m}}\right)$ and showed that below the thermocline the vertical velocity associated to those terms is one to two order of magnitude smaller than the one associated to the deformation of the flow. An exception is the study of (Qiu et al. 2020) in which vertical velocities arising from diffusive terms remain of magnitude comparable to those produced by ageostrophic deformation $\left(\mathbf{Q}_{\mathrm{twag}}\right)$ well below the mixed layer. We suspect that this is because vertical velocities due to mixing are not calculated explicitly in Qiu et al. (2020) but instead are obtained as a residual and thus also contain contributions from various sources such as imperfect boundary conditions and other numerical errors (see Sec. 4c and 4d).

We will limit ourselves to comparing the two $\omega$ reconstructions that are most commonly used with real ocean data: the QG version where $\omega_{Q G}$ is solely forced by the curvature of the 
geostrophic flow $\left(\mathbf{Q}_{\mathbf{Q G}}=2 \mathbf{Q}_{\mathbf{t w g}}\right)$ as in Hoskins et al. (1978); and a more complete formulation (NG) in which the $\mathbf{Q}$ vector forcing $\omega_{N G}$ includes some contribution from the ageostrophic flow $\left(\mathbf{Q}_{\mathrm{NG}}=2 \mathbf{Q}_{\mathrm{tw}}+\mathbf{Q}_{\mathrm{dag}}\right)$.

The $\mathbf{Q}_{\mathbf{t w}_{\mathbf{g}}}$ vector in the $\mathrm{QG}$ formulation has the form:

$$
\mathbf{Q}_{\mathbf{t w}_{\mathbf{g}}}=\frac{g}{\rho_{0}}\left(\frac{\partial u_{g}}{\partial x} \frac{\partial \rho}{\partial x}+\frac{\partial v_{g}}{\partial x} \frac{\partial \rho}{\partial y}, \frac{\partial v_{g}}{\partial y} \frac{\partial \rho}{\partial y}+\frac{\partial u_{g}}{\partial y} \frac{\partial \rho}{\partial x}\right)
$$

where $u_{g}$ and $v_{g}$ are the horizontal components of the geostrophic velocity. In this study, these velocities are estimated by applying the thermal wind balance downward starting from the sea level with the reference velocities at that level being derived from the model sea level anomalies. This procedure mimics what can be optimally done with real oceanic data, assuming that sea level elevation is known with good accuracy.

The NG formulation takes into account the curvature of the total flow and the deformation of the ageostrophic flow,

$$
\begin{aligned}
\mathbf{Q}_{\mathrm{tw}} & =\frac{g}{\rho_{0}}\left(\frac{\partial u}{\partial x} \frac{\partial \rho}{\partial x}+\frac{\partial v}{\partial x} \frac{\partial \rho}{\partial y}, \frac{\partial v}{\partial y} \frac{\partial \rho}{\partial y}+\frac{\partial u}{\partial y} \frac{\partial \rho}{\partial x}\right) \\
\mathbf{Q}_{\text {dag }} & =f\left(\frac{\partial v}{\partial x} \frac{\partial u_{a g}}{\partial z}-\frac{\partial u}{\partial x} \frac{\partial v_{a g}}{\partial z}, \frac{\partial v}{\partial y} \frac{\partial u_{a g}}{\partial z}-\frac{\partial u}{\partial y} \frac{\partial v_{a g}}{\partial z}\right)
\end{aligned}
$$

where $u_{a g}$ and $v_{a g}$ are the ageostrophic horizontal velocities. In practice, $u_{a g}$ and $v_{a g}$ are obtained as the difference between model horizontal velocities and the calculated geostrophic velocities $u_{g}$ and $v_{g}$.

Two baseline reconstructions $\omega_{Q G}$ and $\omega_{N G}$ are thus computed from the adiabatic QG and NG formulations of the $\mathbf{Q}$ vector. For each of them the computation is made on the horizontal subdomains defined in section $2 \mathrm{a}$. The domain extension in the vertical goes from the surface down to $1600 \mathrm{~m}$. Dirichlet boundary conditions are used at all the frontiers. A tridimensional buoyancy frequency, $\mathrm{N}^{2}$, that varies both in the horizontal and in the vertical is used to solve all the inversions, although tests were run using a horizontally averaged profile and little differences were 
observed (not shown). Specific reconstructions using different resolution, domain size or boundary conditions are made to explore sensitivities and described in the corresponding sections.

To investigate the relative importance of different sources of errors we will carry out two forms of omega reconstruction with either perfect right-hand side ( $\omega$ will be denoted $\omega_{\dagger}$ ) or perfect boundary conditions ( $\omega$ will be denoted $\omega^{*}$ ). Perfect rhs omega inversions are computed using forcing terms that are derived from model vertical velocities and the lhs of (9):

$$
\nabla \cdot \mathbf{Q}_{\dagger}=f^{2} \frac{\partial^{2} w_{\text {model }}}{\partial z^{2}}+\nabla_{h}\left(N^{2} \cdot \nabla_{h} w_{\text {model }}\right) .
$$

Precisely, (14) is evaluated using second order centered differences, i.e. in a way that is consistent with how the MUDPACK elliptic solver (Adams 1989) that we use is being implemented. For instance, along the $\mathrm{x}$ direction we use:

$$
\left.\frac{\partial^{2} w}{\partial x^{2}}\right|_{i}=\frac{1}{\Delta x}\left[\frac{\left(w_{i+1}-w_{i}\right)}{\Delta x}-\frac{\left(w_{i}-w_{i-1}\right)}{\Delta x}\right]
$$

Alternatively perfect boundary conditions can be imposed by applying $w_{\text {model }}$ at the edges of the inversion domain. Note that this is however not precisely possible to do at the ocean surface because it is a moving interface in NEMO while it must be held fixed in the solving of the omega equation.

\section{c. Baroclinic mode decomposition}

Under the assumptions of flat bottom and rigid lid at the ocean surface the linearized primitive (or quasi-geostrophic) equations governing the horizontal and vertical components of inviscid fluid motion can be separated (Cushman-Roisin and Beckers 2011). In the vertical, two sets of normal mode eigenfunctions $F_{n}$ and $G_{n}$ form complete bases onto which pressure/horizontal velocity and vertical velocities can respectively be projected (see annex A1). 
Normal mode decomposition has proved useful even in situations where all above assumptions are not satisfied and in particular when the bottom of the ocean is not flat (e.g. Rocha et al. 2013). In order to gain insight into the sources of reconstruction errors normal modes decomposition will be used to interpret the differences in reconstruction skills for the different regimes.

In practice the MODES program available at http://www.d.umn.edu/ smkelly/software.html (Kelly 2016) is used to determine the mode structure at each model point. The modal amplitude of the model vertical velocity is then determined locally.

\section{Description of the vertical velocity regimes}

The four regions and two seasons selected for this study exhibit contrasted dynamical regimes. This will allow us to explore the sensitivity of the omega reconstruction behaviour and skills to the nature of the meso/submesoscale turbulence that produces the vertical velocities. The diversity of vertical flow behavior is visually illustrated by snapshots of model vertical velocities (Figs. 1, 2, $3,4,5)$ and confirmed by their spectral distribution of variance (Fig. 6a,b).

In the LMX region, the displayed vertical section was chosen so as to cross the Gulf Stream (Fig. 2). In this region the EKE is about ten times higher than in the other regions (Fig. 7c,d), the root-mean-square (rms) of $w$ is 3 to 5 times higher (Fig. 7e,f) and intense density fronts are observed both in June and December (Fig. 2). In the frontal region, the vertical velocity structures extend down to $1500 \mathrm{~m}$ (Fig. 7e,f) depth or more, with peak $|w|$ in excess of $80 \mathrm{~m} \mathrm{day}^{-1}$. Overall, this is the region with the largest vertical velocity variance at all scales and a dominant fraction of this variance is found at the largest scales fitting in the study domain. Also note that LMX interior vertical velocities are only weakly affected by the seasonal cycle of the near-surface submesoscale activity (which is present but hardly visible in Fig. 1). 
Compared to the LMX region, the AZO region is a lot less energetic (Fig. 7c,d); the isopycns are flatter; and the horizontal velocities are slower. The vertical circulation is by far the weakest of all four regions (Fig. 7e, f and 6) and exhibits limited seasonal changes. Vertical velocities are organized into structures whose size is intermediate (smaller than in LMX but larger than in OSM and to a lesser extent REK) as readily apparent from the inspection of fields in the physical space and also from the spectral distribution of $w$ variance (Fig. 6): in the range of scales larger than 10 $\mathrm{km}$ the $w$ spectrum is flatter (resp. steeper) than that for LMX (resp. REK and OSM). $w$ patterns in Fig. 3 also tend to be tilted with respect to the vertical axis. This is a plausible indication that vertically propagating IG waves are involved in the generation of $w$ (slanted phase lines associated with near-inertial waves are found in numerous studies, e.g., Furuichi et al. 2008).

In the REK region, the average vertical velocity magnitude is $O\left(10 \mathrm{~m} \mathrm{day}^{-1}\right)$ with localized higher values near mesoscale structures that can reach of up to $100 \mathrm{~m} \mathrm{day}^{-1}$ and tend to have a large vertical extension (Fig. 1 and 4). In the physical space, $w$ structures are frequently tilted with respect to the vertical although less so than in AZO. Again this is presumably the signature of near-inertial wave activity. But contrary to the AZO case, the horizontal patterns visible in $w$ fields are consistent with the structuring role of the (sub)mesoscale activity particularly during summer:presence of multipolar $w$ patterns (Lapeyre and Klein 2006b; Viúdez 2018), that could be the signature of eddy-induced Ekman pumping and/or vortex Rossby waves (Mcwilliams et al. 2003; Buongiorno Nardelli 2013; Barceló-Llull et al. 2017), as in the left corner of Fig. 1c; elongated filaments of elevated $w$ (Capet et al. 2016). During winter the enhancement of mixed layer submesoscale turbulence is particularly marked (Fig. 1c). Most of the fine-scale $w$ patterns appear to be confined into the mixed layer and the visual aspect of the $w$ field differs noticeably on either side of the mixed layer base. On the other hand, $w$ increases significantly in magnitude from summer to winter (Fig. 7e,f). In terms of spatial scales summer $w$ have a clear mesoscale domi- 
nance with a peak around 50-100 km wavelength. For winter $w$, the role of the mesoscale is less prominent while the contribution associated with submesoscales is strongly reinforced (Fig. 6).

Vertical velocities in the OSM region share many similarities with those of REK: magnitude is $O\left(10 \mathrm{~m} \mathrm{day}^{-1}\right)$; flatness of the $w$ power spectrum in the mesoscale range $50-100 \mathrm{~km}$; importance of the near-surface submesoscales particularly during the wintertime; modest indication of vertical tilt. The main difference between the two regions is the greater degree of $w$ continuity across the mixed layer and upper thermocline in winter, presumably as a consequence of very low upper ocean stratification in the OSM region (Fig. 7b).

\section{Omega reconstruction of the vertical velocity fields}

\section{a. Baseline skill assessment}

The skills of the omega reconstructions vary greatly depending on the region and season as illustrated qualitatively (resp. as quantified) in Figs. 2-5 (resp. in Fig. 8). Fig. 8 represents the spectral coherence between the model $w$ and reconstructed $\omega$, i.e., the degree of co-variance between them as a function of scale. The scales and wavelengths above which the spectral coherence is larger than 0.6 for the QG and NG inversion are listed in table 1 along with the fraction of $w$ variance retained at scales larger than that threshold.

In general, omega reconstructions perform best at scales $\gtrsim 10-20 \mathrm{~km}$. The energetic mesoscale structures are better reproduced and spectral coherence drops rapidly at scales $\lesssim 2-10 \mathrm{~km}$, i.e., the omega reconstruction are not well-suited in the submesoscale range. This is particularly well illustrated by the LMX region where spectral coherence reaches levels close to 1 above $20 \mathrm{~km}$ scale but drops below 0.6 around $7-8 \mathrm{~km}$ (Table 1). On the other hand, there is relatively limited $w$ variance at fine-scale. Thus, the overall quality of the reconstructions is manifest for the randomly 
chosen situation shown in Fig. 2 where both the intensity and lateral/vertical extension of the $w$ poles are well reproduced. Overall, $80-90 \%$ of the $w$ signal is captured with a coherence of 0.6 or higher in LMX.

Wintertime OSM is a notable exception where coherence levels remain elevated down to $3 \mathrm{~km}$ scales, particularly when ageostrophic effects are accounted for (see below). As in LMX, upper ocean turbulent stirring and ageostrophic secondary circulations reach down to depths of $300-500$ $\mathrm{m}$ in this region but the deformation radius is much smaller than in LMX and the stratification is particularly weak. The $w$ field is thus organised in fine-scale structures that are frequently several hundred meters thick in the vertical (Fig. 5), many of which are well captured by the omega reconstruction.

In the REK region, the omega equation reproduces adequately some of the stronger and larger vertical velocity structures organized in alternated bands of upward and downward velocity intensity (Fig. 4). As for the finer slanted structures visible in both seasons but more intense in winter they are almost completely missed. The spectral coherence peaks for scales of $\sim 10 \mathrm{~km}$ with lower coherence for smaller and also larger scales. This might arise because vertical velocities at large scale are driven by other dynamical processes associated with the terms not retained in our omega reconstructions such as Ekman and inertial pumping.

Finally, in agreement with the visual impression drawn from Fig. 3, omega reconstructions perform very poorly for wintertime AZO with spectral coherence systematically below 0.5 . Including ageostrophic effects has virtually no impact on the reconstruction skill for the AZO region as clearly illustrated in Fig. 3.

More generally, including the ageostrophic terms makes a significant difference for the LMX and wintertime OSM reconstruction only (the scale where the 0.6 coherence threshold is found decreases by up to $20 \%$ for these cases). We relate this to the fact that high Rossby number 
dynamics is prevalent in both regions albeit for different reasons. In the LMX sectors, the frontality of the Gulf Stream leads to intense turbulent stirring by the mesoscale field. In the OSM sector deep mixed layers in winter lead to intense submesoscale activity whose influence reaches to great depths owing to the reduced subsurface stratification. Conditions resembling those found for winter OSM also exist in the REK region in winter but, in this latter case, the modest stratification present around $200 \mathrm{~m}$ depth (Fig. 7b) seems sufficient to isolate the interior circulation from the influence of the near-surface dynamics and thwart ageostrophic effects, hence the limited increase in coherence between $\omega_{Q G}$ and $\omega_{N G}$ in Fig. 8 .

\section{b. Sensitivity to temporal averaging}

An important limitation of the omega reconstructions presented in the previous section may arise from the role played by IG waves. This role cannot be captured by the forcing terms we retained for the expression of $\mathbf{Q}$ in the $\mathrm{QG}$ and $\mathrm{NG}$ reconstructions which ignores the material rate of change of the thermal wind imbalance $\left(\mathbf{Q}_{\mathbf{d r}}\right.$ in 10$)$. Using daily averages tends to reduce the effects of these processes, particularly where the inertial period is close to $24 \mathrm{~h}$ (at $30^{\circ}$ latitude), but does not eliminate them. It is unclear whether the IG wave and subinertial quasi-balanced flow contributions to $w$ should be separated (see discussion section) but the behavior of the omega reconstruction as a function of the temporal scale content of the forcing term $\nabla \cdot \mathbf{Q}$ is of practical as well as theoretical interest. This behavior is explored by increasing the degree of temporal averaging that is performed prior to the computation of the omega reconstruction right-hand side. All simulated fields are averaged similarly, including $w_{\text {model }}$. The expectation is that more temporal averaging will reduce the amount of variance associated with the $w$ field to be reconstructed but improve the skill of the omega reconstruction for that low-passed $w$. The existence of such a trade-off is confirmed in Fig. 9 for most dynamical regimes. Fig. 9 presents the spectral coherence 
between $\omega_{N G}$ and $w_{\text {model }}$ for larger $(10-25 \mathrm{~km})$ and finer $(3-10 \mathrm{~km})$ scales as a function of the remaining variance ratio $\left.\operatorname{Var}(<w\rangle_{t}\right) / \operatorname{Var}(w)$, where $\left.<\cdot\right\rangle_{t}$ is the low-pass temporal averaging whose length is varied from 1 day (standard output for that study) and 10 days. Results are shown for $\omega_{N G}$ but only minor differences were found with $\omega_{Q G}$.

For all regions except LMX an optimum in spectral coherence is found for time-averaging longer than 1 day, both for the large scales $(10-25 \mathrm{~km})$ and the fine scales $(3-10 \mathrm{~km})$. The averaging period associated with this optimum is between 2 and 5 days. As anticipated, spectral coherence improvement comes at the expense of a loss of reconstructed $w$ variance. In several cases the loss of $w$ variance is less than 25\%: REK, AZO and OSM for the large scales and REK for the fine scales during summer; OSM for the large scales during winter. Particularly for this latter case the spectral coherence improvement is substantial and 2-5 day averaging seems justified. Conversely, the most dramatic improvement in $\left(\omega_{N G}, w\right)$ spectral coherence seen in the AZO region (winter, $10-25 \mathrm{~km}$ scales, 5-day averaging) has a major effect on the retained $\omega_{N G}$ variance (only $37 \%$ of the original $w$ variance) and may be of limited utility in practice.

\section{c. Discretization errors}

We also wish to draw the reader's attention on the delicacies of the omega inversion and the implications these delicacies have on the estimation of vertical velocities. To do so we attempt to minimize reconstruction errors. In addition to imposing perfect boundary conditions by applying $w_{\text {model }}$ at the edges of our domain, we also compute a "perfect" right-hand side $\nabla . \mathbf{Q}_{\dagger}$ to be used in the elliptic inversion (see section $2 \mathrm{~b}$ ). The resulting vertical velocity estimate $\omega_{*}^{*}$ found as a numerical solution is associated with minute errors, on average $3 \%$ with the solving parameter choices we pass to MUDPACK. These errors result from 1) the imperfect convergence of the elliptic solver and 2) the imperfect boundary condition at the ocean surface. 
To gain insight into the sensitivity of the elliptic inversion, variant $\omega_{\ddagger}^{*}$ were also computed by employing a discretization scheme alternative to (15) to calculate $\nabla_{h}^{2} w_{\text {model }}$ in (14):

$$
\left.\frac{\partial^{2} w}{\partial x^{2}}\right|_{i}=\frac{1}{2 \Delta x}\left[\frac{\left(w_{i+2}-w_{i}\right)}{2 \Delta x}-\frac{\left(w_{i}-w_{i-2}\right)}{2 \Delta x}\right]
$$

Both schemes are consistent, centered, and second order in accuracy. They only differ by the length of their stencil ( 3 or 5 points).

We find that the discretization choice has a moderate but significant impact. In the LMX region in June for instance the use of the scheme (16) to compute the "perfect" rhs produces relative $w$ errors in excess of $12 \%$, to be compared with the minimal convergence errors of $\sim 3 \%$ with scheme (15). The elevated error figure obtained with the second scheme helps place realistic limits to vertical velocity estimation attempts and draws the attention on the fact that minute errors/uncertainties on the rhs of the omega equation can have significant impacts on the reconstructed vertical velocities.

\section{d. Sensitivity and errors due to boundary conditions}

Finally, an important source of error when solving the omega equation is the choice of the bottom boundary condition. As numerous studies have pointed out (Allen and Smeed 1996; Rudnick 1996; Pascual et al. 2004) $\omega$ is not very dependant on the lateral boundary conditions but the form of the BBC and the depth where it is applied can have a strong impact on the solution of the equation (Pidcock et al. 2012; Rudnick 1996). Two types of BBCs are generally used, the Dirichlet condition $w=0$ or the Neumann condition $\partial w / \partial z=0$. A third one was proposed by Rudnick (1996): to limit the influence of the boundary condition on the solution, the vertical grid was extended below the depth where observations were available. Over the depth range with no observations $\nabla . \mathbf{Q}$ was simply assumed to be zero. In practice, this led Rudnick (1996) to shift 
the bottom boundary from $400 \mathrm{~m}$ (where the deepest observation was available) to $2524 \mathrm{~m}$ depth where a Dirichlet BBC $(w=0)$ was imposed.

To assess the impact of the BBC on the solution we compare the errors obtained for Dirichlet and Neumann conditions and for different domain extensions, with the bottom boundary depth $z_{\text {bottom }}$ ranging from $150 \mathrm{~m}$ to $1600 \mathrm{~m}$ (Fig. 10). This is done in the four regions and for the two seasons. We also test the boundary condition proposed by Rudnick (1996) with two different depths (400 $\mathrm{m}$ and $800 \mathrm{~m}$ ) below which $\nabla . \mathbf{Q}$ is assumed to be zero down to $800 \mathrm{~m}$ and $1600 \mathrm{~m}$ respectively. In this section, the omega reconstruction is solved using $\nabla . \mathbf{Q}_{\dagger}$ forcing computed as described in section $2 \mathrm{~b}$ so relative errors exceeding a few percents can only be due to boundary conditions.

Figure 10 shows the relative errors of omega reconstructions made at the reference depths $z_{a}$ when different boundary conditions are applied. The errors at depth $z_{a}$ are horizontally and temporally averaged over the entire reconstruction domain on the horizontal and over the 11 consecutive daily model outputs. The relative error field can be quite large, generally in excess of 0.5 for domain depths shallower than $500 \mathrm{~m}$, despite a good agreement in $w$ and $\omega$ patterns. This is generally consistent with the findings of several past studies (Pinot et al. 1996; Allen et al. 2001; Rixen et al. 2003).

Relative errors varies with $z_{\text {bottom }}$ in a relatively simple and intuitive way: deeper $z_{\text {bottom }}$ systematically translates into weaker errors with Neumann or Dirichlet boundary condition. More precisely, errors tend to stabilize around $20 \%$ when $z_{\text {bottom }}$ greater than $1000-1500$ meters are used (Table 2), with the notable exception of REK for which relative errors are $\sim 0.3-0.4$ and still decreasing at $z_{\text {bottom }}=1600 \mathrm{~m}$. The most evident manifestation of error saturation behavior is found for LMX and Neumann boundary conditions, with a threshold around $z_{\text {bottom }}=700 \mathrm{~m}$ beyond which little improvement is observed. More generally we note that: the quality of the reconstruction can vary from low to high depending on the choice being made for the depth of the 
boundary condition; except in the LMX region the Neumann and Dirichlet boundary conditions give relatively similar results; the use of a buffer region to increase the domain depth following Rudnick (1996) can ameliorate the reconstruction skill and provide an optimum over all possible choices but the improvement is generally marginal. Estimating the relative error as a volumeaverage over the whole water volume above the depth where the boundary condition is applied paints a more complicated picture. For instance, in a number of situations, reconstruction errors are found to increase when the location of the boundary layer deepens beyond certain thresholds (not shown). We relate this to the fact that deep vertical velocities can be particularly intense owing to processes not properly resolved by our implementations of the omega reconstructions (IG wave activity, flow-topography interaction).

In an attempt to gain further insight, we use vertical mode decomposition to characterize the vertical structure of the $w$ field. Consider a situation where $w$ would project onto a single mode, for instance baroclinic mode 2. In that case, $w$ systematically vanishes at the zero crossing of $\mathrm{G}_{2}$ and a Dirichlet boundary condition at that depth would not introduce any error. A perfect Neumann boundary condition would similarly exist at the zero crossings of $F_{2}$. There are several potential obstacles preventing this from happening. Most importantly, vertical velocity tends to be associated with fine horizontal scales and this tends to be also true in the vertical (Fig. 3-5). The vertical mode decomposition of $w$ generally involves many modes, and in most cases, less than half of the $w$ signal is explained by the sum of the first three vertical modes (Fig. 12, annex A1). It is therefore impossible to choose a fixed depth where the dominant $\mathrm{F}_{n}$ or $\mathrm{G}_{n}$ are zeros. The LMX regime is an exception where the $w$ vertical structure projects to a large extent onto the first three baroclinic modes (Fig. 12). For LMX the smallest reconstruction errors (volume averaged) are obtained for a Neumann boundary condition placed at approximately $750 \mathrm{~m}$ depth (not shown), i.e., the depth below which errors evaluated at a single depth $\left(z_{a}=220 \mathrm{~m}\right)$ tend to reach a plateau 
around $0.2-0.25$ (Fig. 10a,e). The $750 \mathrm{~m}$ value falls in the depth range where $\mathrm{F}_{1}$ and $\mathrm{F}_{3}$ are very close to zero (Fig. 11a). The link between the vertical mode's structure and the effect of $z_{\text {bottom }}$ on the reconstruction skill is far less clear for the other three regions. We attribute these inconsistencies to the limited role played by the gravest baroclinic modes in AZO, REK and OSM (Fig 12).

To better characterize the reconstruction errors due to imperfect (bottom) boundary conditions, we compute an alternative omega reconstruction $\omega_{N G}^{*}$ using perfect $w_{\text {model }}$ information at the boundaries (see section 2b). Differences between $\omega_{N G}^{*}$ and $w_{\text {model }}$, can only arise from the simplification made to $\mathbf{Q}$ in the NG inversion (that is, considering $\mathbf{Q}_{\mathbf{d r}}, \mathbf{Q}_{\mathrm{th}}$ and $\mathbf{Q}_{\mathbf{d m}}$ as null). They range from $\sim 1$ to $\sim 5 \mathrm{~m} \mathrm{day}^{-1}$ depending on the region and season. They are typically one to three times as high as errors associated with a Dirichlet boundary condition at $1600 \mathrm{~m}$ (Table 2) and generally lower than the ones associated with a Dirichlet boundary condition at $500 \mathrm{~m}$ (Fig. 10). Discrepancies in spectral distribution between $\omega_{N G}^{*}$ and $w_{\text {model }}$ are much reduced compared to those for $\omega_{N G}$. This is particularly true for the scales below $1 \mathrm{~km}$ (Fig. 6c and 6d). A similar skill improvement is noticeable for the spectral coherence (Fig. 8). A significant fraction of the fine-scale vertical motions in the upper ocean is thus directly linked to unidentified processes active below $1600 \mathrm{~m}$. Consistent with previous studies (e.g. see Jouanno et al. 2016) flow-topography interactions is a likely candidate, which would pose serious difficulties if it were to be explicitly incorporated into the omega reconstruction approach (inversion would need to be performed for the whole ocean depth range with the knowledge of the vertical velocity at the ocean floor $w=-\mathbf{u} \nabla h$ ). We do not see any prospect for this but this source of errors should be kept in mind. 


\section{Discussion}

Since the 1980s a large number of studies have focused on inferring oceanic vertical velocities through more or less elaborate forms of the omega equation. Most of them have been applied to local in-situ data, in which the sources of errors are difficult to identify and quantify (see the bibliography table SI1). In some instances the reconstructed vertical velocity field has been used to qualitatively interpret concomitant tracer distributions (Pollard and Regier 1992; Rudnick 1996; Martin and Richards 2001; Allen et al. 2005; Ruiz et al. 2009; Pallàs-Sanz et al. 2010; Rousselet et al. 2019). The uncertainty on reconstructed $w$ is an important limitation when doing so (also note that the vertical tracer distribution at any given time reflects the past history of vertical advection - and mixing). $w$ velocities are now increasingly being used quantitatively, e.g., as inputs to tracer models (Pascual et al. 2015; Barceló-Llull et al. 2016).

Model studies have addressed the various sources of errors involved and the ways to reduce their impact with a general focus on three main issues: i) the merits of more complete versions of the omega equation; ii) the sensitivity to particular choices of boundary conditions when solving the omega equation; iii) the errors induced by the lack of resolution, homogeneity, and synopticity of in situ sampling when the omega reconstruction is applied to real ocean observations (see SI1). These model-based assessments of $\omega$ reconstructions were typically performed in simplified flow conditions, composed of a single coherent eddy or front, with a marked preference for the early stages of destabilisation of baroclinic zones (Strass 1994; Pinot et al. 1996; Allen et al. 2001; Rixen et al. 2003; Viúdez 2018). More recently, the omega equation has been used to determine oceanic vertical velocity fields over larger domains and extended time periods from observationbased gridded reconstructions of temperature and salinity fields (Pascual et al. 2015; Barceló-Llull et al. 2016; Buongiorno Nardelli et al. 2018). The resulting $\omega$ fields can subsequently be used 
to estimate vertical fluxes and this has been attempted for several biogeochemical tracers, over different relevant time scales.

Overall, a general assessment of the skills and limitations of the omega reconstruction is lacking. The present study is an effort in that direction that mainly pertains to i) and ii) and we deliberately excluded errors of the type iii). Although they pose important and perhaps leading-order limitations to w reconstructions from observations, this type of errors could be significantly reduced by observing the ocean at higher resolution in specific regions of interest and combining these observations with fine-scale remotely sensed information and model integrations. Optimal ways to produce such combinations may involve relocation strategies as proposed in Rixen et al. (2001) and Pascual et al. (2004). Errors i) and ii) pose different type of challenges that have not received much attention. We have followed the steps of Strass (1994) whose analyses of the omega reconstruction skills included a scale-dependant coherence diagnostic. An originality of our study is to estimate the fidelity of $w$ reconstructions for fully turbulent realistic flows. Several findings reported in the previous section deserve further discussion.

\section{On the limited skills of omega reconstructions at fine-scale}

First, the important limitations to the omega reconstructions found at relatively fine-scale, despite the absence of type iii) errors may seem surprising. Some remarks are thus in order. An important motivation for this work is the representation of vertical tracer fluxes between the surface and interior of the ocean. Therefore we focused on vertical velocities tens of meters below the mixed layer. At such depths, vertical velocity spectra are significantly more red than closer to the surface where whiter spectra have been reported (Ponte and Klein 2013; Klein et al. 2008). This means that modest levels of variance remain in the scale range where coherence has dropped 
down. Conversely, a large fraction of $w$ variance ( $>60 \%$ for all regions and seasons) is found in the scale range where coherence is above 0.6 (table 1).

This being said, the coherence drop off at scales below $10 \mathrm{~km}$ is interesting in itself and deserves further discussion. The only regime for which significant skill is being obtained into submesoscale range is winter OSM, which clearly stands out in terms of coherence (Fig. 8). This is also the only regime for which the influence of surface intensified frontal turbulence is found to penetrate down to the depth of analysis $\left(z_{a}=380 \mathrm{~m}\right)$, owing to extremely weak subsurface stratifications. This regime is also the one for which the NG reconstruction provides the largest improvement over the QG reconstruction. The lack of reconstruction skills generally found at submesoscale is therefore not due to a particular difficulty at capturing vertical velocities associated with frontal turbulence. Instead, we attribute reconstruction errors to the imperfection of the $\mathrm{BBC}$ used to invert the elliptic omega equation and to the neglect of fluctuations in thermal wind imbalance due to partly or fully unbalanced fast processes such as inertial gravity waves. The former source of error is identified and quantified explicitly while the importance of the latter is inferred indirectly. Further elaboration on these two error sources follow.

The boundary conditions impact omega reconstructions over the entire study domain owing to the elliptic nature of the omega equation. It has been known for a long time that the solution can be improved by properly choosing the type and location of the BBC. We explored the possibility that the BBC strategies could be rationalised based on vertical mode decompositions. Although one exception was found in the Gulf Stream region, vertical velocities tend to project onto a large number of vertical modes (Fig. 12) and no ideal positioning of the BBC can be chosen a priori. Pushing the BBC position $z_{\text {bottom }}$ toward greater depths leads to reconstruction improvements even beyond $1500 \mathrm{~m}$. This is not feasible when observations are used to perform omega reconstructions and the typical choices made in this situation $\left(z_{\text {bottom }} \sim 500 \mathrm{~m}\right.$ see review table SI1) fall in the range 
where errors are most sensitive to $z_{\text {bottom }}$. In the Gulf stream region, vertical velocities associated with upper ocean frontal dynamics extend deep into the water column and tend to project mainly onto the first 3 vertical modes (Fig. 12). These properties are qualitatively consistent with the fact that, for the LMX domain, the Neumann BBC outperforms the Dirichlet BBC and that the relative error curve as a function of BBC depth levels off for $z_{\text {bottom }}$ below $\sim 700 \mathrm{~m}$. Similar behaviors may presumably be observed in other western boundary regions and the Antarctic Circumpolar Current region. Finally, note that, given a depth down to which data is available to feed an omega reconstruction, the so-called telescopic method developed by Rudnick provides at best a marginal improvement over the classical Dirichlet or Neumann boundary conditions.

The presence of inertia-gravity waves near and beyond the drop-off scale is not accounted for as a source of $w$ (in our case, neglecting the forcing term $\mathbf{Q}_{\mathbf{d r}}$ was due to limitations on the model outputs at our disposal but including this term for real ocean situations would pose extremely stringent if not impractical requirements on sampling strategies). The AZO region is the one where inertia-gravity waves are suspected to play the largest role, if only because balanced turbulence is very weak there. There may be other more subtle consequences of the inertia-gravity wave activity on the omega reconstruction we have presented. For instance, REK has more $w$ variance in winter but coherence between $\omega_{N G}$ and $w_{\text {model }}$ is degraded at that season compared to summer. Although seasonal changes appear comparatively unimportant in the LMX region, a slight coherence degradation occurs in winter: winter coherence for the NG reconstruction is $\sim 0.1$ lower than in summer over the scale range $2-8 \mathrm{~km}$ (gaps are smaller at larger scale but the degradation is noticeable up to $20 \mathrm{~km}$ ). Again, signs of enhanced NIW activity for the winter analysis period compared to the summer one suggest that near-inertial waves are responsible for this degradation.

For completeness, two other possible sources of omega reconstruction errors are listed here. Daily averaged variables are used to compute the forcing terms which are nonlinear combinations 
of these variables. This means that Reynold-type forcing terms should formally be included due to rapid (intra-day) fluctuations in $u, v$ and $\rho$. Kinetic energy corresponding to motions with temporal scales below 1 day is very low in simulations with $d x \sim 2 \mathrm{~km}$ and we have assumed that this is a small effect. It would need to be verified that the same applies when in-situ observations are low-passed filtered prior to computing rhs terms for a real ocean omega reconstruction. Forcing terms associated with mixing of momentum and buoyancy have also been ignored. This is generally considered as a valid approximation sufficiently far from the mixed layer while this source of error is expected to increase at shallower depth (see below for more elaboration on this) in the context of ocean tracer dynamics. Although generally consistent with our findings the latest results of Qiu et al. (2020) cast some doubts on the smallness of $w$-forcing by buoyancy and momentum diffusion. In a western boundary context, this term is found to be comparable to $w$-forcing by ageostrophic kinematic deformation and stronger than $w$-forcing by thermal wind imbalance deformation or thermal wind imbalance tendency, even well into the ocean interior (see their figure 4). This issue would however need to be scrutinized based on an explicit computation of the mixed effect on $w$. Qiu et al. (2020) infer this term as a residual which therefore lumps together various contributions including that due to BBC errors. Model output limitations have not allowed us to undertake this.

\section{On the consequences of limited reconstruction skills at fine-scales for tracer dynamics}

Ultimately, what matters in most applications is not vertical velocity per se but the vertical transport of oceanic properties, for instance upward fluxes of dissolved iron enriching the euphotic layer in the Southern Ocean (Nicholson et al. 2019), or downward fluxes of organic material filling the ocean interior and having key implications on $\mathrm{O} 2$ consumption and distribution (Boyd et al. 2019). Vertical tracer fluxes arise from vertical motions because upward and downward motions 
are, on average, associated with distinct tracer values. We find it useful to discuss three types of limit cases in which this can occur and where the limitations of the omega reconstruction described above may be more or less problematic depending on the scenario. To this end, we consider a nutrient-like tracer $\tau$ whose concentration gradient is directed downward. The same reasoning would apply to tracer with an upward gradient (e.g., phytoplankton or dissolved oxygen).

An important distinction between two types of limit cases can be made depending whether baroclinic instability (BCI) plays an important role or not.

\section{$\underline{\text { Case } 1}$}

When $\mathrm{BCI}$ is important, turbulent vertical motions are strongly constrained by the fact that $\left\langle w^{\prime} b^{\prime}\right\rangle>0$, where $b$ refers to buoyancy, $\langle$.$\rangle is a low-pass averaging operator and the prime notation$ refers to deviations from this average. $\mathrm{BCI}$ can thus contribute to transport a tracer $\tau$ vertically provided that the distributions of $\tau$ and $b$ are correlated. Far enough from the surface in the ocean interior available potential energy (APE) release is mainly achieved through mesoscale velocities (Lapeyre et al. 2006; Capet et al. 2016). Unless the correlations of $\tau$ and $b$ were weak at mesoscale and strong at submesoscale, the proper description of mesoscale $w$ would thus suffice to capture the dominant part of $\left\langle w^{\prime} \tau^{\prime}\right\rangle$. Typically, correlations between a tracer $\tau$ and buoyancy exist owing to processes acting at large-scale, although large decorrelation can ensue from biological reactions (e.g. for oxygen and nitrate). Correlations are then transferred down to smaller scales by turbulent cascades and decorrelation between buoyancy and $\tau$ is preferentially found at the finest scales (see discussion in Fox-Kemper et al. 2013, and references therein). Therefore, we expect omega reconstructions to be useful in this case despite the limitations we have described.

We now turn to the situation where vertical motions are not constrained by baroclinic instability and result instead from, e.g., fossil mesoscale/submesoscale turbulence and/or internal wave activity. In this situation, any water parcel has a well defined reference depth position $z_{\text {ref }}$ (quite 
independent of horizontal position) around which it may oscillate and an additional process must be invoked for these oscillations to produce $\tau$ vertical/diapycnal fluxes. We take a Lagrangian viewpoint and consider $\delta z=\int_{t} w d t$, the vertical displacement of a water parcel from its equilibrium position and we distinguish two limit cases illustrated in Fig. 13.

\section{Case 2}

Let us consider the situation in which a sink term for $\tau$ is located in the euphotic layer where photosynthesis draws $\tau$ levels down (Fig. $13 \mathrm{a}$ and b). The $\tau$ anomalies are schematically represented for a mesoscale (panel a) and a submesoscale (panel b) upward oscillation where the amplitude of the oscillations have been chosen so as to reflect the distribution of $w$ variance found in the ocean interior for all regimes $\left(\delta z^{m}>\delta z^{s}\right)$. Because $\delta z^{m}>\delta z^{s}$ and also because the mesoscale excursions last on average longer the photosynthesis sink term for $\tau$ is more effective at mesoscale than it is at submesoscale. The tracer biogeochemistry acts as a low-pass filter and a representation of fine-scale vertical motions is unessential in the determination of tracer vertical fluxes. The deficiencies of the omega equation evidenced in this study should thus have limited consequences on the determination of tracer vertical fluxes, e.g., as performed in Barceló-Llull et al. (2016).

Note that the depth where the mean gradient of $\tau$ resides is a parameter that is potentially important to consider. If this mean gradient were located very close below the mixed layer base, the region where the photosynthesis sink term is active would be closer and the relative amplitude of submesoscale vertical motions (compared to that for the mesoscale) would be larger, hence a stronger influence of submesoscale processes.

\section{Case 3}

The last limit case is the one where the only possible modification of the tracer concentration attached to water parcels is through (vertical/diapycnal) mixing with surrounding water masses when and where the underlying flow structure produces local shear enhancement. Shear being 
proportional to spatial derivates of the velocities, fine-scale vertical motions can potentially have a major contribution on $\tau$ vertical/diapycnal fluxes despite their lesser magnitude relative to those at mesoscale. Reconstructed vertical velocities have a general bias toward overly weak variance that is particularly pronounced at scales below 30 to $50 \mathrm{~km}$, depending on the regime. This bias and its impact on the determination of tracer fluxes may be amenable to statistical corrections if omega reconstructions for case 3 type problems turn out to be of importance. The impact of (very) fine-scale motions on tracer dynamics is presently accounted for by shear-driven mixing parametrizations (Gregg 1989; Polzin et al. 1995 and references therein) which represent the effect of internal gravity wave. There are evidences that different forms of heterogeneities such as submesoscale flows affect internal wave breaking (Whitt and Thomas 2013; Avicola et al. 2007) but the utility of the omega equation to incorporate these effects in our ocean representations is presently unclear.

\section{Conclusions}

The ever expanding coverage of observational networks supplemented by satellite observations with increasing spatio-temporal resolution like the upcoming SWOT mission (d' Ovidio et al. 2019) was a strong motivation for this work. In the coming years we may be able to estimate vertical fluxes more accurately and at finer scale than ever before. Today, the most common tool to infer vertical velocity is the omega equation. In this paper we explored the ability of the most common configurations adopted to solve this equation to provide information on the vertical circulation at different horizontal length scales. The main novelty of our work is to have used a broad variety of regimes which helped unravel the diversity of reasons underlying the errors in omega reconstructions of $w$ fields, depending on the regional dynamics. 
Generally the adiabatic omega reconstruction gives good results for mesoscale vertical circulation, typically for structures with horizontal scales larger than $10 \mathrm{~km}$. These structures tend to be associated with a large part of the $w$ variance, although their relative importance and the precise skills of the omega reconstruction at such scales depend on the local dynamics. Omega reconstruction skills degrade strongly in the submesoscale range. This degradation is manifest both in terms of reduced variance levels and lack of coherence when comparing reconstructed and true vertical velocities. There are two main reasons explaining these findings: the overall weakness of (true) $w$ variance levels below the surface boundary layer in the submesoscale range; the coexistence in that range of several processes contributing to vertical motions, not just frontal turbulence but also inertia-gravity waves which force vertical velocities through terms that are generally not accessible. Overall, the best reconstructions are thus observed in conditions characterised by energetic turbulence and/or weak stratification (such that near-surface frontal processes are felt well into the ocean interior). In particular we observed the best results in regimes for a western boundary (LMX with elevated EKE) and wintertime Porcupine Abyssal Plain (OMS, very weak water column stratification). Conversely the weak vertical flow found in the AZO region where EKE is low and subsurface stratification is relatively strong is poorly captured by the omega approach.

Implications on the role of submesoscale vertical velocities and whether/how to integrate them into our representations of property exchanges in the ocean interior will require further elaboration. Numerous studies have highlighted the strong impact frontal submesoscale turbulence can have on physical and biogeochemical fluxes (Lapeyre et al. 2006; Klein et al. 2008; Thomas and Ferrari 2008; Capet et al. 2008). For a given tracer $\tau$, this is only true to the extent that the mean distribution of $\tau$ exhibits vertical contrasts sufficiently close to the surface where frontal activity remains important. How close depends on the background environment and in particular on the 
upper ocean stratification. The Surface Quasi-Geostrophic (SQG) theory is useful to qualitatively apprehend this dependance (Lapeyre et al. 2006).

In most past studies dealing with vertical velocities a quasi geostrophic version of the omega equation was used, but more recently, several authors have included higher order terms. For this reason, we also evaluated the possible improvements obtained by using a more elaborate version of the adiabatic equation compared to the simple quasi geostrophic formulation. NG reconstructions that include ageostrophic forcing terms due to flow deformation leads to improved reconstructions. The improvements are substantial only in conditions where near-surface frontal processes (i.e., submesoscales) are important. This finding has important implications in the context of efforts aimed at estimating vertical velocities in the real ocean because the adiabatic QG reconstruction only requires the knowledge of the density field and of the sea surface height (or a reference level of no motion).

The consequences of various numerical choices on the reconstruction skills have been investigated in this work. Choices made for the bottom boundary condition have a major impact. Pushing the boundary condition as deep as possible is the only rule of thumb that can be provided. The relative performance of the Dirichlet versus Neumann boundary conditions cannot be anticipated a priori while the BBC tweak proposed by Rudnick (1996) offers at best marginal improvements. Obviously, omega reconstruction skills will worsen when it is applied to real observations with lesser spatial resolution (for the ocean interior data), synopticity issues and instrumental errors.

\section{APPENDIX}




\section{A1. Baroclinic mode decomposition}

To support the results in section $4 \mathrm{~d}$, we use a normal mode decomposition to establish possible relations between the depth of the vertical boundary condition and the error committed when estimated $w$ with $\omega$. The eigenfunctions are solution of the eigenvalues problem:

$$
\frac{\partial^{2} G_{n}}{\partial z^{2}}+\frac{N^{2}}{c_{n}^{2}} G_{n}=0
$$

or

$$
\frac{\partial}{\partial z}\left(\frac{1}{N^{2}} \frac{\partial F_{n}}{\partial z}\right)+\frac{1}{c_{n}^{2}} F_{n}=0
$$

with the boundary conditions:

$$
G_{n}=0 \text { at } z=0 \text { and } z=-H
$$

$$
\frac{\partial F_{n}}{\partial z}=0 \text { at } z=0 \text { and } z=-H
$$

where the vertical velocity modes $G_{n}$ and the pressure modes $F_{n}$ are related through the relation,

$$
F_{n}=\frac{\partial G_{n}}{\partial z} .
$$

Vertical variability can then be projected onto the vertical modes $F_{n}$ and $G_{n}$ so that,

$$
\begin{aligned}
p(x, y, z, t) & =\sum_{n=0}^{\infty} \tilde{p}_{n}(x, y, t) F_{n}(z) \\
w(x, y, z, t) & =\sum_{n=0}^{\infty} \tilde{w}_{n}(x, y, t) G_{n}(z),
\end{aligned}
$$

with $\tilde{p}_{n}$ and $\tilde{w}_{n}$ the modal amplitudes of the pressure and vertical velocity respectively.

To investigate more thoroughly the contribution of the vertical modes to the vertical velocity signal, we expressed the vertical velocity by the projection on the three firsts vertical modes and a residue,

$$
w(x, y, z, t)=\sum_{n=1}^{N} \tilde{w}_{n}(x, y, t) G_{n}(z)+\varepsilon .
$$


$\varepsilon$ was estimated for $N=[1: 3]$. The number of profiles where $\varepsilon / w$ was lower than 0.5 , that is to say that more than half of the $w$ dynamics is explained by the first one, two or three vertical modes, was estimated (Fig. 12).

Note that in Figure 11, $\mathrm{F}_{n}$ and $\mathrm{G}_{n}$ are calculated for a mean stratification that is obtained using spatial averaging over the entire region and time averaging over 11 daily model outputs. However they do not differ substantially from the modes calculated on each point and used to infer modal amplitudes (Sec. 2c).

Acknowledgments. This work has been partly supported by the NASA/CNES Tosca project BIOSWOT. Alice Pietri was supported by a CNES postdoctoral grant. The authors wish to thanks the SWOT cal/val team for providing an stimulating environment in the development of our study. The NATL60 simulation can be accessed at https://meom-group.github.io/swotnat160/access-data.html. The authors wish to thank two anonymous reviewers for their comments and suggestions for improving the paper.

\section{References}

Adams, J. C., 1989: mudpack: Multigrid portable fortran software for the efficient solution of linear elliptic partial differential equations. Applied Mathematics and Computation, 34 (2), 113 146, doi:10.1016/0096-3003(89)90010-6.

Allen, J. T., and D. Smeed, 1996: Potential Vorticity and Vertical Velocity at the Iceland-Faeroes Front. Journal of Physical Oceanography, 26, 2611-2634.

Allen, J. T., D. A. Smeed, A. J. G. Nurser, J. W. Zhang, and M. Rixen, 2001: Diagnosis of vertical velocities with the $\mathrm{QG}$ omega equation: A relocation method to obtain pseudo-synoptic 
data sets. Deep-Sea Research Part I: Oceanographic Research Papers, 48 (6), 1347-1373, doi: 10.1016/S0967-0637(00)00085-6.

Allen, J. T., and Coauthors, 2005: Diatom carbon export enhanced by silicate upwelling in the northeast Atlantic. Nature, 437, 1-5, doi:10.1038/nature03948.

Amores, A., G. Jordà, T. Arsouze, and J. Le Sommer, 2018: Up to What Extent Can We Characterize Ocean Eddies Using Present-Day Gridded Altimetric Products? Journal of Geophysical Research: Oceans, 123 (10), 7220-7236, doi:10.1029/2018JC014140.

Avicola, G. S., J. N. Moum, A. Perlin, and M. D. Levine, 2007: Enhanced turbulence due to the superposition of internal gravity waves and a coastal upwelling jet. Journal of Geophysical Research: Oceans, 112 (6), 1-20, doi:10.1029/2006JC003831.

Balwada, D., K. S. Smith, and R. Abernathey, 2018: Submesoscale Vertical Velocities Enhance Tracer Subduction in an Idealized Antarctic Circumpolar Current. Geophysical Research Letters, 45 (18), 9790-9802, doi:10.1029/2018GL079244.

Barceló-Llull, B., E. Mason, A. Capet, and A. Pascual, 2016: Impact of vertical and horizontal advection on nutrient distribution in the southeast Pacific. Ocean Science, 12 (4), 1003-1011, doi:10.5194/os-12-1003-2016.

Barceló-Llull, B., E. Pallàs-Sanz, P. Sangrà, A. Martínez-Marrero, S. N. Estrada-Allis, and J. Arístegui, 2017: Ageostrophic Secondary Circulation in a Subtropical Intrathermocline Eddy. Journal of Physical Oceanography, 47 (5), 1107-1123, doi:10.1175/jpo-d-16-0235.1.

Barceló-Llull, B., A. Pascual, E. Mason, and S. Mulet, 2018: Comparing a Multivariate Global Ocean State Estimate With High-Resolution in Situ Data: An Anticyclonic Intrathermocline 
Eddy Near the Canary Islands. Frontiers in Marine Science, 5, 1-15, doi:10.3389/fmars.2018. 00066.

Bower, A., and T. Rossby, 1989: Evidence of Cross-Frontal Exchange Processes in the Gulf Stream Based on Isopycnal RAFOS Float Data. Journal of Physical Oceanography, 19, 1177 1190.

Boyd, P. W., H. Claustre, M. Levy, D. A. Siegel, and T. Weber, 2019: Multi-faceted particle pumps drive carbon sequestration in the ocean. Nature, 568, 327-335, doi:10.1038/ s41586-019-1098-2.

Buckingham, C. E., and Coauthors, 2016: Seasonality of submesoscale flows in the ocean surface boundary layer. Geophysical Research Letters, 43, 2118-2126.

Buongiorno Nardelli, B., 2013: Vortex waves and vertical motion in a mesoscale cyclonic eddy. Journal of Geophysical Research: Oceans, 118 (10), 5609-5624, doi:10.1002/jgrc.20345.

Buongiorno Nardelli, B., S. Guinehut, A. Pascual, Y. Drillet, S. Ruiz, and S. Mulet, 2012: Towards high resolution mapping of 3-d mesoscale dynamics from observations. Ocean Science, $8,885-$ 901, doi:10.5194/os-8-885-2012.

Buongiorno Nardelli, B., S. Mulet, and D. Iudicone, 2018: Three-Dimensional Ageostrophic Motion and Water Mass Subduction in the Southern Ocean. Journal of Geophysical Research: Oceans, 123 (2), 1533-1562, doi:10.1002/2017JC013316.

Buongiorno Nardelli, B., and R. Santoleri, 2005: Methods for the reconstruction of vertical profiles from surface data: Multivariate analyses, residual GEM, and variable temporal signals in the North Pacific Ocean. Journal of Atmospheric and Oceanic Technology, 22 (11), 1762-1781, doi:10.1175/JTECH1792.1. 
Buongiorno Nardelli, B., 2020: A multi-year time series of observation-based 3D horizontal and vertical quasi-geostrophic global ocean currents. Earth System Science Data, 12(3), 1711-1723, doi:10.5194/essd-12-1711-2020.

Callies, J., and R. Ferrari, 2018: Baroclinic instability in the presence of convection. Journal of Physical Oceanography, 48, 45-60.

Capet, X., P. Klein, B. L. Hua, G. Lapeyre, and J. C. McWilliams, 2008: Surface kinetic energy transfer in surface quasi-geostrophic flows. Journal of Fluid Mechanics, 604, 165-174, doi: $10.1017 / \mathrm{S} 0022112008001110$

Capet, X., G. Roullet, P. Klein, and G. Maze, 2016: Intensification of Upper-Ocean Submesoscale Turbulence through Charney Baroclinic Instability. Journal of Physical Oceanography, 46 (11), 3365-3384, doi:10.1175/jpo-d-16-0050.1.

Chenillat, F., B. Blanke, N. Grima, P. J. S. Franks, X. Capet, and P. Rivière, 2015: Quantifying tracer dynamics in moving fluids: a combined Eulerian-Lagrangian approach. Frontiers in Environmental Science, 3 (June), 1-15, doi:10.3389/fenvs.2015.00043.

Cushman-Roisin, B., and J.-M. Beckers, 2011: Introduction to Geophysical Fluid Dynamics, Vol. 101. 2nd ed., Academic Press, 875 pp.

d' Ovidio, F., and Coauthors, 2019: Frontiers in fine-scale in situ studies: Opportunities during the SWOT fast sampling phase. Frontiers in Marine Science, 6, doi:10.3389/fmars.2019.00168.

D’Asaro, E. A., K. B. Winters, and R. C. Lien, 2004: Lagrangian estimates of diapycnal mixing in a simulated K-H instability. Journal of Atmospheric and Oceanic Technology, 21 (5), 799-809, doi:10.1175/1520-0426(2004)021<0799:LEODMI $\rangle 2.0 . C O ; 2$. 
Davies-Jones, R., 1991: The frontogenetical forcing of secondary circulations. part i: The duality and generalization of the q vector. Journal of the Atmospheric Sciences, 48 (4), 497-509, doi: 10.1175/1520-0469(1991)048<0497:TFFOSC $\rangle 2.0 . C O ; 2$.

Ducousso, N., J. Le Sommer, J. M. Molines, and M. Bell, 2017: Impact of the Symmetric Instability of the Computational Kind at mesoscale- and submesoscale-permitting resolutions. Ocean Modelling, 120 (October), 18-26, doi:10.1016/j.ocemod.2017.10.006.

Ferrari, R., 2011: A frontal challenge for climate models. Science, 332 (6027), 316-317, doi: 10.1126/science.1203632.

Fox-Kemper, B., R. Lumpkin, and F. O. Bryan, 2013: Lateral transport in the ocean interior. Ocean Circulation and Climate: A 21st century perspective, G. Siedler, S. M. Griffies, J. Gould, and J. A. Church, Eds., Academic Press (Elsevier Online), International Geophysics, Vol. 103, 185209.

Fresnay, S., A. L. Ponte, S. Le Gentil, and J. Le Sommer, 2018: Reconstruction of the 3-D Dynamics From Surface Variables in a High-Resolution Simulation of North Atlantic. Journal of Geophysical Research: Oceans, 123 (3), 1612-1630, doi:10.1002/2017JC013400.

Furuichi, N., T. Hibiya, and Y. Niwa, 2008: Model-predicted distribution of wind-induced internal wave energy in the world's oceans. Journal of Geophysical Research: Oceans, 113 (9), 1-13, doi:10.1029/2008JC004768.

Giordani, H., and G. Caniaux, 2001: Sensitivity of cyclogenesis to sea surface temperature in the Northwestern Atlantic. Monthly Weather Review, 129 (6), 1273-1295, doi:10.1175/ 1520-0493(2001)129〈1273:SOCTSS $\rangle 2.0 . C O ; 2$. 
Giordani, H., G. Caniaux, and L. Prieur, 2005: A simplified 3D oceanic model assimilating geostrophic currents: Application to the POMME experiment. Journal of Physical Oceanography, 35 (5), 628-644, doi:10.1175/JPO2724.1.

Giordani, H., and S. Planton, 2000: Modeling and Analysis of Ageostrophic Circulation over the Azores Oceanic Front during the SEMAPHORE Experiment. Monthly Weather Review, 128 (7), 2270-2287, doi:10.1175/1520-0493(2000)128〈2270:maaoac $\rangle 2.0 . c o ; 2$.

Giordani, H., L. Prieur, and G. Caniaux, 2006: Advanced insights into sources of vertical velocity in the ocean. Ocean Dynamics, 56 (5-6), 513-524, doi:10.1007/s10236-005-0050-1.

Gregg, M. C., 1989: Scaling turbulent dissipation in the thermocline. Journal of Geophysical Research, 94 (C7), 9686-9698, doi:10.1029/jc094ic07p09686.

Guinehut, S., A. L. Dhomps, G. Larnicol, and P. Y. Le Traon 2012: High resolution 3-D temperature and salinity fields derived from in situ and satellite observations. Ocean Science, $\mathbf{8 ( 5 ) ,}$ 845-857, doi:10.5194/os-8-845-2012.

Hamlington, P. E., L. P. Van Roekel, B. Fox-Kemper, K. Julien, and G. P. Chini, 2014: Langmuirsubmesoscale interactions: Descriptive analysis of multiscale frontal spindown simulations. Journal of Physical Oceanography, 44, 2249-2272.

Helber, R. W., and R. H. Weisberg, 2001: Equatorial upwelling in the western pacific warm pool. Journal of Geophysical Research, 106, 8989-9003.

Held, I. M., R. T. Pierrehumbert, S. T. Garner, and K. L. Swanson, 1995: Surface quasi-geostrophic dynamics. J. Fluid Mech., 282, 1-20, doi:10.1017/S0022112095000012. 
Horii, T., Y. Masumoto, I. Ueki, S. P. Kumar, and K. Mizuno, 2011: Intraseasonal vertical velocity variation caused by the equatorial wave in the central equatorial indian ocean. Journal of Geophysical Research, 116.

Hoskins, B. J., 1982: the Mathematical theory of frontogenesis. Ann. Rev. Fluid Mech, 15 (12), $131-151$.

Hoskins, B. J., I. Draghici, and H. C. Davies, 1978: A new look at the $\omega$ equation. Quarterly Journal of the Royal Meteorological Society, 104 (439), 31-38, doi:10.1002/qj.49710443903.

Isern-Fontanet, J., B. Chapron, G. Lapeyre, and P. Klein, 2006: Potential use of microwave sea surface temperatures for the estimation of ocean currents. Geophysical Research Letters, 33 (24), 1-5, doi:10.1029/2006GL027801.

Jouanno, J., X. Capet, G. Madec, G. Roullet, and P. Klein, 2016: Dissipation of the energy imparted by mid-latitude storms in the Southern Ocean. Ocean Science, 12 (3), 743-769, doi: 10.5194/os-12-743-2016.

Joyce, T. M., J. M. Toole, P. Klein, and L. N. Thomas, 2013: A near-inertial mode observed within a Gulf Stream warm-core ring. Journal of Geophysical Research: Oceans, 118 (4), 1797-1806, doi:10.1002/jgrc.20141.

Kelly, S. M., 2016: The Vertical Mode Decomposition of Surface and Internal Tides in the Presence of a Free Surface and Arbitrary Topography. Journal of Physical Oceanography, 46 (12), 3777-3788, doi:10.1175/jpo-d-16-0131.1.

Klein, P., B. L. Hua, G. Lapeyre, X. Capet, S. Le Gentil, and H. Sasaki, 2008: Upper Ocean Turbulence from High-Resolution 3D Simulations. Journal of Physical Oceanography, 38 (8), 1748-1763, doi:10.1175/2007jpo3773.1. 
Klein, P., J. Isern-Fontanet, G. Lapeyre, G. Roullet, E. Danioux, B. Chapron, S. Le Gentil, and H. Sasaki, 2009: Diagnosis of vertical velocities in the upper ocean from high resolution sea surface height. Geophysical Research Letters, 36 (12), 1-5, doi:10.1029/2009gl038359.

LaCasce, J. H., and A. Mahadevan, 2006: Estimating subsurface horizontal and vertical velocities from sea-surface temperature. Journal of Marine Research, 64, 695-721, doi:10.1357/ 002224006779367267.

Lapeyre, G., 2009: What Vertical Mode Does the Altimeter Reflect? On the Decomposition in Baroclinic Modes and on a Surface-Trapped Mode. Journal of Physical Oceanography, 39 (11), 2857-2874, doi:10.1175/2009jpo3968.1.

Lapeyre, G., and P. Klein, 2006a: Dynamics of the Upper Oceanic Layers in Terms of Surface Quasigeostrophy Theory. Journal of Physical Oceanography, 36 (2), 165-176, doi:10.1175/ jpo2840.1.

Lapeyre, G., and P. Klein, 2006b: Impact of the small-scale elongated filaments on the oceanic vertical pump. Journal of Marine Research, 64 (6), 835-851, doi:10.1357/002224006779698369.

Lapeyre, G., P. Klein, and B. L. Hua, 2006: Oceanic Restratification Forced by Surface Frontogenesis. Journal of Physical Oceanography, 36 (8), 1577-1590, doi:10.1175/jpo2923.1.

Le Sommer, J., E. P. Chassignet, and A. J. Wallcraft, 2018: Ocean Circulation Modeling for Operational Oceanography: Current Status and Future Challenges. New Frontiers in Operational Oceanography, E. Chassignet, A. Pascual, J. Tintoré, and J. Verron, Eds., GODAE OceanView, 289-306, doi:10.17125/gov2018.ch12. 
Leach, H., 1987: The diagnosis of synoptic-scale vertical motion in the seasonal thermocline. Deep Sea Research Part A, Oceanographic Research Papers, 34 (12), 2005-2017, doi:10.1016/ 0198-0149(87)90095-1.

Ledwell, J. R., D. J. McGillicuddy, and L. A. Anderson, 2008: Nutrient flux into an intense deep chlorophyll layer in a mode-water eddy. Deep-Sea Research Part II: Topical Studies in Oceanography, 55 (10-13), 1139-1160, doi:10.1016/j.dsr2.2008.02.005.

Lévy, M., R. Ferrari, P. J. Franks, A. P. Martin, and P. Rivière, 2012a: Bringing physics to life at the submesoscale. Geophysical Research Letters, 39 (14), 1-14, doi:10.1029/2012GL052756.

Lévy, M., P. J. Franks, and K. S. Smith, 2018: The role of submesoscale currents in structuring marine ecosystems. Nature communications, 9 (1), 4758, doi:10.1038/s41467-018-07059-3.

Lévy, M., O. Jahn, S. Dutkiewicz, and M. J. Follows, 2014: Phytoplankton diversity and community structure affected by oceanic dispersal and mesoscale turbulence. Limnology and Oceanography: Fluids and Environments, 4 (1), 67-84, doi:10.1215/21573689-2768549.

Lévy, M., P. Klein, A. Tréguier, D. Iovino, G. Madec, S. Masson, and K. Takahashi, 2010: Modifications of gyre circulation by sub-mesoscale physics. Ocean Modelling, 34 (1-2), 1-15, doi: 10.1016/j.ocemod.2010.04.001.

Lévy, M., L. Resplandy, P. Klein, G. Madec, A.-M. Trguier, S. Masson, and K. Takahashi, 2012b: Large-scale impacts of submesoscale dynamics on phytoplankton: Local and remote effects. Ocean Modelling. Ocean Modelling, 43-44, 77-93, doi:10.1016/j.ocemod.2011.12.003.

Lien, R.-C., and Coauthors, 2014: The LatMix Summer Campaign: Submesoscale Stirring in the Upper Ocean. Bulletin of the American Meteorological Society, 96 (8), 1257-1279, doi: 10.1175/bams-d-14-00015.1. 
Lindstrom, S. S., X. Qian, and D. R. Watts, 1997: Vertical motion in the gulf stream and its relation to meanders. Journal of Geophysical Research: Oceans, 102, 8485-8503.

Lindstrom, S. S., and R. D. Watts, 1994: Vertical motion in the gulf stream near 68 w. Journal of Physical Oceanography, 24, 2321-2333.

Martin, A. P., and K. J. Richards, 2001: Mechanisms for vertical nutrient transport within a North Atlantic mesoscale eddy. Deep-Sea Research Part II: Topical Studies in Oceanography, 48 (45), 757-773, doi:10.1016/S0967-0645(00)00096-5.

Mcwilliams, J. C., L. P. Graves, and M. T. Montgomery, 2003: A formal theory for vortex rossbywaves and vortex evolution. Geophysical \& Astrophysical Fluid Dynamics, 97, 275-309, doi: 10.1080/0309192031000108698.

Nagai, T., A. Tandon, and D. L. Rudnick, 2006: Two-dimensional ageostrophic secondary circulation at ocean fronts due to vertical mixing and large-scale deformation. Journal of Geophysical Research: Oceans, 111 (9), 1-18, doi:10.1029/2005JC002964.

Nicholson, S. A., M. Lévy, J. Jouanno, X. Capet, S. Swart, and P. M. Monteiro, 2019: Iron Supply Pathways Between the Surface and Subsurface Waters of the Southern Ocean: From Winter Entrainment to Summer Storms. Geophysical Research Letters, 46 (24), 14 567-14 575.

Pallàs-Sanz, E., T. M. Johnston, and D. L. Rudnick, 2010: Frontal dynamics in a California Current System shallow front: 2. Mesoscale vertical velocity. Journal of Geophysical Research: Oceans, 115 (12), C12 068, doi:10.1029/2010JC006474.

Pascual, A., D. Gomis, R. L. Haney, and S. Ruiz, 2004: A Quasigeostrophic Analysis of a Meander in the Palamós Canyon: Vertical Velocity, Geopotential Tendency, and a Relocation Tech- 
nique. Journal of Physical Oceanography, 34 (10), 2274-2287, doi:10.1175/1520-0485(2004) 034〈2274:aqaoam $\rangle 2.0 . c 0 ; 2$.

Pascual, A., S. Ruiz, B. Buongiorno Nardelli, S. Guinehut, D. Iudicone, and J. Tintoré, 2015: Net primary production in the Gulf Stream sustained by quasi-geostrophic vertical exchanges. Geophysical Research Letters, 42 (2), 441-449, doi:10.1002/2014GL062569.

Pascual, A., and Coauthors, 2017: A multiplatform experiment to unravel meso- and submesoscale processes in an intense front (alborex). Frontiers in Marine Science, 4, doi:10.3389/fmars.2017. 00039.

Pauley, P. M., and S. J. Nieman, 1992: A comparison of quasigeostrophic and nonquasigeostrophic vertical motions for a model-simulated rapidly intensifying marine extratropical cyclone. Monthly Weather Review, 120 (7), 1108-1134, doi:10.1175/1520-0493(1992)120<1108: ACOQAN $>2.0 . \mathrm{CO} ; 2$.

Pidcock, R., A. Martin, J. Allen, S. C. Painter, and D. Smeed, 2012: The spatial variability of vertical velocity in an Iceland basin eddy dipole. Deep Sea Research Part I: Oceanographic Research Papers, 72, 121-140, doi:10.1016/j.dsr.2012.10.008.

Pinot, J.-M., J. Tintoré, and D.-P. Wang, 1996: A study of the omega equation for diagnosing vertical motions at ocean fronts. Journal of Marine Research, 54 (2), 239-259, doi:10.1357/ 0022240963213358.

Pollard, R. T., and L. A. Regier, 1992: Vorticity and Vertical Circulation at an Ocean Front. Journal of Physical Oceanography, 22 (6), 609-625, doi:10.1175/1520-0485(1992)022/0609: vavcaa $\rangle 2.0 . c 0 ; 2$. 
Polzin, K. L., J. M. Toole, and R. W. Schmitt, 1995: Finescale parameterizations of turbulent dissipation. Journal of Physical Oceanography, 25 (3), 306-328, doi:10.1175/1520-0485(1995)

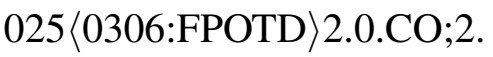

Ponte, A. L., and P. Klein, 2013: Reconstruction of the upper ocean 3D dynamics from high-resolution sea surface height. Ocean Dynamics, 63 (7), 777-791, doi:10.1007/ s10236-013-0611-7.

Qiu, B., S. Chen, P. Klein, H. Torres, J. Wang, L. L. Fu, and D. Menemenlis, 2020: Reconstructing upper ocean vertical velocity field from sea surface height in the presence of unbalanced motion. Journal of Physical Oceanography, 49 (10), 55-79, doi:10.1175/JPO-D-19-0172.1.

Qiu, B., S. Chen, P. Klein, J. Wang, H. Torres, L.-L. Fu, and D. Menemenlis, 2018: Seasonality in transition scale from balanced to unbalanced motions in the world ocean. Journal of Physical Oceanography, 48, 591-605, doi:10.1175/JPO-D-17-0169.1.

Rixen, M., J. T. Allen, R. T. Pollard, and J.-M. Beckers, 2003: Along or across front ocean survey strategy? The estimation of quasi-geostrophic vertical velocities and temperature fluxes. Geophysical Research Letters, 30 (5), n/a-n/a, doi:10.1029/2002gl015810.

Rixen, M., J. Beckers, and J. T. Allen, 2001: Diagnosis of vertical velocities with the QG Omega equation : a relocation method to obtain pseudo-synoptic data sets. Deep Sea Research Part I: Oceanographic Research Papers, 48, 1347-1373.

Rocha, C. B., A. Tandon, I. C. Da Silveira, and J. A. M. Lima, 2013: Traditional quasi-geostrophic modes and surface quasi-geostrophic solutions in the Southwestern Atlantic. Journal of Geophysical Research: Oceans, 118 (5), 2734-2745, doi:10.1002/jgrc.20214. 
Rousselet, L., and Coauthors, 2019: Vertical Motions and Their Effects on a Biogeochemical Tracer in a Cyclonic Structure Finely Observed in the Ligurian Sea. Journal of Geophysical Research: Oceans, 1-14, doi:10.1029/2018JC014392.

Rudnick, D. L., 1996: Inferring the geostrophic and vertical velocity fields. J. of Geophys. Res., 101 (C7), 16,216-291,303.

Ruiz, S., A. Pascual, B. Garau, I. Pujol, and J. Tintoré, 2009: Vertical motion in the upper ocean from glider and altimetry data. Geophysical Research Letters, 36 (14), 1-6, doi: 10.1029/2009GL038569.

Shcherbina, A. Y., E. A. D’Asaro, C. M. Lee, J. M. Klymak, M. J. Molemaker, and J. C. McWilliams, 2013: Statistics of vertical vorticity, divergence, and strain in a developed submesoscale turbulence field. Geophysical Research Letters, 40, 4706-4711.

Shearman, R. K., J. A. Barth, J. S. Allen, and R. L. Haney, 2000: Diagnosis of the threedimensional circulation in mesoscale features with large rossby number. Journal of Physical Oceanography, 30 (11), 2687-2709, doi:10.1175/1520-0485(2001)031〈2687:DOTTDC〉2. $0 . \mathrm{CO} ; 2$.

Siegelman, L., P. Klein, P. Rivire, A. F. Thompson, H. S. Torres, M. Flexas, and D. Menemenlis 2020: Enhanced upward heat transport at deep submesoscale ocean fronts. Nature Geoscience, 13, 50-55, doi:10.1038/s41561-019-0489-1.

Smith, K. M., P. E. Hamlington, and B. Fox-Kemper, 2016: Effects of submesoscale turbulence on ocean tracers. Journal of Geophysical Research: Oceans, 121, 908-933. 
Soufflet, Y., P. Marchesiello, F. Lemarié, J. Jouanno, X. Capet, L. Debreu, and R. Benshila, 2016: On effective resolution in ocean models. Ocean Modelling, 98, 36-50, doi:10.1016/j.ocemod. 2015.12.004.

Steffen, E. L., and E. A. D’Asaro, 2002: Deep Convection in the Labrador Sea as Observed by Lagrangian Floats. Journal of Physical Oceanography, 32 (2), 475-492, doi:10.1175/ 1520-0485(2002)032〈0475:dcitls $\rangle 2.0 . c o ; 2$.

Strass, V. H., 1994: Mesoscale Instability and Upwelling. Part 2: Testing the Diagnostics of Vertical Motion with a Three-Dimensional Ocean Front Model. Journal of Physical Oceanography, 24 (8), 1759-1767, doi:10.1175/1520-0485(1994)024〈1759:miaupt $\rangle 2.0 . c o ; 2$.

Su, Z.,H. S. Torres, P. Klein, A. F. Thompson, L. Siegelman, J. Wang, D. Menemenlis, and C. Hill, 2020: High-Frequency Submesoscale Motions Enhance the Upward Vertical Heat Transport in the Global Ocean. Journal of Geophysical Research: Oceans, 9, 1-13, doi: 10.1029/2020JC016544.

Sullivan, P. P., and J. C. McWilliams, 2018: Frontogenesis and frontal arrest of a dense filament in the oceanic surface boundary layer. Journal of Fluid Mechanics, 837, 341-380.

Suzuki, N., B. Fox-Kemper, P. E. Hamlington, and L. P. Van Roekel, 2016: Surface waves affect frontogenesis. Journal of Geophysical Research: Oceans, 121, 3597-3624.

Thomas, L., and R. Ferrari, 2008: Friction, Frontogenesis, and the Stratification of the Surface Mixed Layer. Journal of Physical Oceanography, 38 (11), 2501-2518, doi:10.1175/ 2008jpo3797.1.

Thomas, L. N., and C. M. Lee, 2005: Intensification of Ocean Fronts by Down-Front Winds. Journal of Physical Oceanography, 35 (6), 1086-1102, doi:10.1175/jpo2737.1. 
Thomas, L. N., C. M. Lee, and Y. Yoshikawa, 2010: The Subpolar Front of the Japan/East Sea. Part II: Inverse Method for Determining the Frontal Vertical Circulation. Journal of Physical Oceanography, 40 (1), 3-25, doi:10.1175/2009jpo4018.1.

Thompson, A. F., A. Lazar, C. Buckingham, A. C. Naveira Garabato, G. M. Damerell, and K. J. Heywood, 2016: Open-Ocean Submesoscale Motions: A Full Seasonal Cycle of Mixed Layer Instabilities from Gliders. Journal of Physical Oceanography, 46 (4), 1285-1307, doi:10.1175/ JPO-D-15-0170.1.

Thomsen, S., T. Kanzow, F. Colas, V. Echevin, G. Krahmann, and A. Engel, 2016: Do submesoscale frontal processes ventilate the oxygen minimum zone off Peru? Geophysical Research Letters, 43 (15), 8133-8142, doi:10.1002/2016gl070548.

Uchida, T., D. Balwada, R. Abernathey, G. McKinley, S. Smith, and M. Lévy, 2019: The Contribution of Submesoscale over Mesoscale Eddy Iron Transport in the Open Southern Ocean Journal of Advances in Modeling Earth Systems, 11 (12), 3934-3958, doi:10.1029/2019MS001805.

Viúdez, A., 2018: Two modes of vertical velocity in subsurface mesoscale eddies. Journal of Geophysical Research: Oceans, 123 (5), 3705-3722, doi:10.1029/2017JC013735.

Viúdez, Á., and D. G. Dritschel, 2004: Potential Vorticity and the Quasigeostrophic and Semigeostrophic MesoscaleVertical Velocity. Journal of Physical Oceanography, 34 (4), 865-887, doi:10.1175/1520-0485(2004)034〈0865:pvatqa $\rangle 2.0 . c o ; 2$.

Viúdez, Á., J. Tintoré, and R. L. Haney, 2002: About the Nature of the Generalized Omega Equation. Journal of the Atmospheric Sciences, 53 (5), 787-795, doi:10.1175/1520-0469(1996) 053<0787:atnotg $\rangle 2.0 . c 0 ; 2$. 
Volkov, D. L., and L.-L. Fu, 2010: On the Reasons for the Formation and Variability of the Azores Current. Journal of Physical Oceanography, 40 (10), 2197-2220, doi:10.1175/2010jpo4326.1.

Volkov, D. L., and L. L. Fu, 2011: Interannual variability of the Azores Current strength and eddy energy in relation to atmospheric forcing. Journal of Geophysical Research: Oceans, 116 (11), doi:10.1029/2011JC007271.

Whitt, D. B., M. Lévy, and J. R. Taylor, 2019: Submesoscales enhance storm-driven vertical mixing of nutrients : insights from a biogeochemical large eddy simulation. Journal of Geophysical Research, doi:10.1029/.

Whitt, D. B., and L. N. Thomas, 2013: Near-Inertial Waves in Strongly Baroclinic Currents. Journal of Physical Oceanography, 43 (4), 706-725, doi:10.1175/jpo-d-12-0132.1.

Xie, L., E. Pallàs-Sanz, Q. Zheng, S. Zhang, X. Zong, X. Yi, and M. Li, 2017: Diagnosis of 3D vertical circulation in the upwelling and frontal zones east of Hainan Island, China. Journal of Physical Oceanography, 47 (4), 755-774, doi:10.1175/JPO-D-16-0192.1.

Yoshikawa, Y., C. M. Lee, and L. N. Thomas, 2012: The Subpolar Front of the Japan/East Sea. Part III: Competing Roles of Frontal Dynamics and Atmospheric Forcing in Driving Ageostrophic Vertical Circulation and Subduction. Journal of Physical Oceanography, 42 (6), 991-1011, doi: 10.1175/jpo-d-11-0154.1.

Yu, X., A. C. Naveira Garabato, A. P. Martin, C. E. Buckingham, L. Brannigan, and Z. Su, 2019: An annual cycle of submesoscale vertical flow and restratification in the upper ocean. Journal of Physical Oceanography, 49, 1439-1461, doi:10.1175/JPO-D-18-0253.1. 


\section{LIST OF TABLES}

Table 1. Statistics derived from the spectral coherence field (fig.8). Note that relating the horizontal scale $L_{0.6}$ to the deformation radius, $R_{d}$, which is $\sim 15 \mathrm{~km}$ in the OSM and REK regions and $\sim 30 \mathrm{~km}$ in the LMX and AZO regions, yields a performance threshold of $0.3 R_{d}$ on average, with a minimum $0.17 R_{d}$ in winter OSM and a maximum $0.49 R_{d}$ in winter REK. In the AZO region in December the squared coherence never exceeds 0.6 thus leading to a fraction of variance null.

Table 2. Averaged errors (in $\mathrm{m} \mathrm{day}^{-1}$ and percentage of $w_{\text {model }}$ ), at depths $z_{a}$, for $\omega$ with perfect right hand side $\left(\omega_{\dagger}\right)$, perfect boundaries $\left(\omega_{N G}^{*}\right)$ and both $\left(\omega_{\uparrow}^{*}\right)$. See section $2 b$ for the detail on the computation of $\omega$. 
TABLE 1. Statistics derived from the spectral coherence field (fig.8). Note that relating the horizontal scale $L_{0.6}$ to the deformation radius, $R_{d}$, which is $\sim 15 \mathrm{~km}$ in the OSM and REK regions and $\sim 30 \mathrm{~km}$ in the LMX and AZO regions, yields a performance threshold of $0.3 R_{d}$ on average, with a minimum $0.17 R_{d}$ in winter OSM and a maximum $0.49 R_{d}$ in winter REK. In the AZO region in December the squared coherence never exceeds 0.6 thus leading to a fraction of variance null.

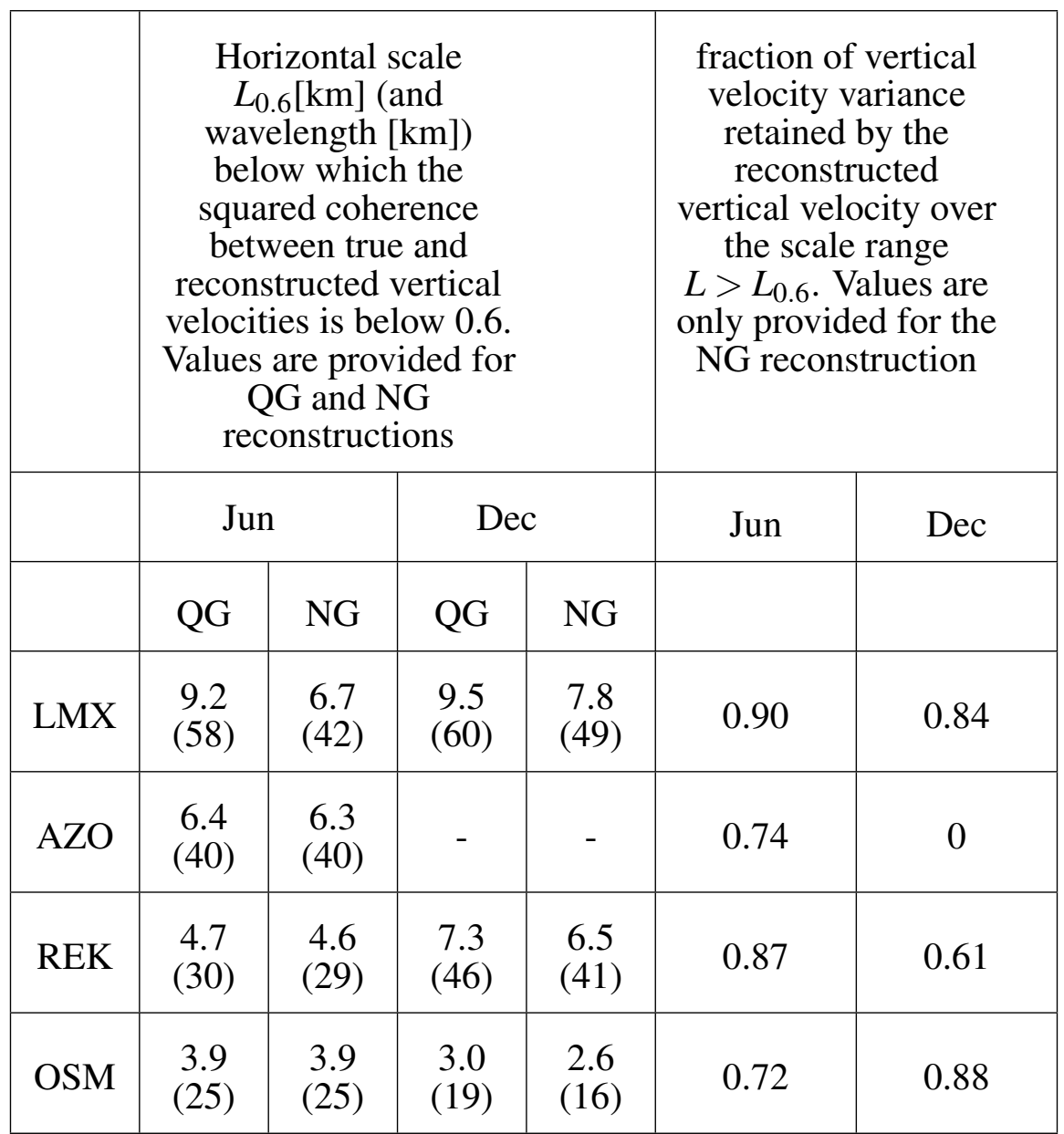


TABLE 2. Averaged errors (in $\mathrm{m} \mathrm{day}^{-1}$ and percentage of $w_{\text {model }}$ ), at depths $z_{a}$, for $\omega$ with perfect right hand ${ }_{1135}$ side $\left(\omega_{\dagger}\right)$, perfect boundaries $\left(\omega_{N G}^{*}\right)$ and both $\left(\omega_{\uparrow}^{*}\right)$. See section $2 \mathrm{~b}$ for the detail on the computation of $\omega$.

\begin{tabular}{|c|c|c|c|c|c|c|}
\hline & \multicolumn{2}{|c|}{$\omega_{\dagger}^{*}$} & \multicolumn{2}{c|}{$\omega_{\dagger}$} & \multicolumn{2}{c|}{$\omega_{N G}^{*}$} \\
\hline LMX & Jun & Dec & Jun & Dec & Jun & Dec \\
\hline & $\begin{array}{c}0.41 \\
(3 \%)\end{array}$ & $\begin{array}{c}0.54 \\
(5 \%)\end{array}$ & $\begin{array}{c}4.08 \\
(28 \%)\end{array}$ & $\begin{array}{c}2.85 \\
(24 \%)\end{array}$ & $\begin{array}{c}4.87 \\
(34 \%)\end{array}$ & $\begin{array}{c}5.21 \\
(44 \%)\end{array}$ \\
\hline AZO & Jun & Dec & Jun & Dec & Jun & Dec \\
\hline & $\begin{array}{c}0.04 \\
(3 \%)\end{array}$ & $\begin{array}{c}0.04 \\
(3 \%)\end{array}$ & $\begin{array}{c}0.25 \\
(20 \%)\end{array}$ & $\begin{array}{c}0.27 \\
(23 \%)\end{array}$ & $\begin{array}{c}0.80 \\
(65 \%)\end{array}$ & $\begin{array}{c}0.95 \\
(79 \%)\end{array}$ \\
\hline REK & Jun & Dec & Jun & Dec & Jun & Dec \\
\hline & $\begin{array}{c}0.13 \\
(4 \%)\end{array}$ & $\begin{array}{c}0.11 \\
(3 \%)\end{array}$ & $\begin{array}{c}0.96 \\
(29 \%)\end{array}$ & $\begin{array}{c}1.19 \\
(29 \%)\end{array}$ & $\begin{array}{c}1.47 \\
(45 \%)\end{array}$ & $\begin{array}{c}2.85 \\
(69 \%)\end{array}$ \\
\hline OSM & Jun & Dec & Jun & Dec & Jun & Dec \\
\hline & $\begin{array}{c}0.06 \\
(2 \%)\end{array}$ & $\begin{array}{c}0.20 \\
(4 \%)\end{array}$ & $\begin{array}{c}0.66 \\
(19 \%)\end{array}$ & $\begin{array}{c}0.90 \\
(16 \%)\end{array}$ & $\begin{array}{c}2.06 \\
(58 \%)\end{array}$ & $\begin{array}{c}2.96 \\
(52 \%)\end{array}$ \\
\hline
\end{tabular}




\section{LIST OF FIGURES}

Fig. 1. Model vertical velocity on 10 june 2008 (left) and 10 december 2008 (right) in the a) LMX region, b) AZO region, c) REK region and d) OSM region. The upper horizontal plan corresponds to the depth of analysis $\left(z_{a}=220 \mathrm{~m}\right.$ in LMX, $250 \mathrm{~m}$ in AZO, and $380 \mathrm{~m}$ in REK and OSM)

Fig. 2. Vertical velocity in the LMX region on 10 June 2008 (left, a, c, e) and 10 December 2008 (right, b, d, f) for the QG inversion (a, b), NG inversion (c, d) and model data (e, f). The thin white (resp. black) lines represent the isopycns (resp. horizontal velocity, $\mathrm{m} \mathrm{s}^{-1}$ ). The bold solid (resp. dashed) black line indicates mixed layer base (resp. the analysis depth $z_{a}$ ).

Fig. 3. As in Fig. 2 but for the AZO region.

Fig. 4. As in Fig. 2 but for the REK region.

Fig. 5. As in Fig. 2 but for the OSM region.

Fig. 6. a,b) Vertical velocity variance power spectra for $w_{\text {model }}$ (solid) and $\omega_{N G}$ (dashed) at depths $z_{a}$ in the LMX (black, $z_{a}=220 \mathrm{~m}$ ), AZO (green, $z_{a}=250 \mathrm{~m}$ ), REK (red, $z_{a}=380 \mathrm{~m}$ ), and OSM (blue, $z_{a}=380 \mathrm{~m}$ ) regions in a) June and b) December. c,d) Same as (a,b) but for $w_{\text {model }}$ (solid) and $\omega_{N G}^{*}$ (dashed, the reconstructed $\omega_{N G}^{*}$ is computed using perfect boundary conditions). Straight (resp. dashed and dot-dashed) lines indicate -2 (resp. $-5 / 3$ and -3 ) roll-offs. The bottom (resp. top) horizontal axis displays the length scale (resp. wavelength).

Fig. 7. Averaged vertical profiles of (a,b) buoyancy frequency, (c,d) eddy kinetic energy and (e,f) vertical velocity root mean square for the LMX (black), AZO (green), REK (red) and OSM (blue) regions in June (upper panel) and December (lower panel). Note the subpanels with a change of scale to accommodate the large differences in EKE between LMX and the other regions.

Fig. 8. a,b) Spectral coherence as a function of scale between $w_{\text {model }}$ and $\omega_{N G}$ (resp. $\omega_{Q G}$ ) at depths $z_{a}$ is represented by a solid (resp. dashed) line in the LMX (black), AZO (green), REK (red), and OSM (blue) regions in a) June and b) December. c,d) Same as (a,b) but the dahed line represent the coherence between $w_{\text {model }}$ and $\omega_{N G}^{*}$ (the reconstructed $\omega_{N G}^{*}$ is computed using perfect boundary conditions). The bottom (resp. top) horizontal axis displays the length scale (resp. wavelength).

Fig. 9. Spectral coherence between $w_{\text {model }}$ and $\omega_{N G}$ at depths $z_{a}$ averaged over the scale ranges (a, b) $10-25 \mathrm{~km}$ and (c, d) $3-10 \mathrm{~km}$ as a function of the fraction of variance retained by each averaging intervals. Results are shown for the LMX (black), AZO (green), REK (red), and OSM (blue) regions in June (left, a, c) and December (right, b, d). The markers represent the timespan of the averages: the star is 1 day, square 2 days, diamond 5 days and triangle 10 days. The highest coherence is indicated by a larger marker with a black contour. The black dashed lines mark a fraction of variance of $75 \%$ and a coherence of 0.6 .

Fig. 10. Relative error, at depths $z_{a}$, between $w_{\text {model }}$ and $\omega_{\dagger}$ in June (a-d) and December (e-h) in the $\operatorname{LMX}(\mathrm{a}, \mathrm{e}), \operatorname{AZO}(\mathrm{b}, \mathrm{f}), \operatorname{REK}(\mathrm{c}, \mathrm{g})$ and OSM $(\mathrm{d}, \mathrm{h})$ regions as a function of the depth where the bottom boundary condition $\left(z_{\text {bottom }}\right.$ in the text) is imposed. Errors for a Dirichlet (resp. Neumann) BBC are represented with a black (resp. blue) line. Black diamonds indicate error values for a Dirichlet boundary condition modified as in Rudnick (1996) (see section $4 \mathrm{~d}$ for details). The vertical bars show the standard deviation over the 11 daily averages used for our analysis. 
Fig. 11. First three baroclinic pressure modes for the a) LMX, b) AZO, c) REK, and d) OSM region in June (red) and December (blue). First three vertical velocity modes for the e) LMX, f) AZO, g) REK, and h) OSM region in June (red) and December (blue). The first mode (resp. 2nd and 3rd modes) is represented with a solid (resp. dashed and dotted) line. The amplitudes of the modes have been scaled so as to vary between -1 and 1 .

Fig. 12. Percentage of vertical water profiles for which the projection of model $w$ on the subset of the gravest vertical modes (1, 2 or 3 gravest modes) leads to an approximation of $w$ with a relative error that is less than $50 \%$, i.e., percentage of profiles where $\varepsilon / w<0.5$ ( $\varepsilon$ being defined in equation A8). Percentages are represented for LMX (black), AZO (green), REK (red) and OSM (blue) in summer (plain lines) and winter (dashed lines) . . . . . . . . . . 70

Fig. 13. schematic representation of the vertical disturbance (y-direction) and associated tracer anomaly temporal evolution undergone by a water parcel in 4 different situations. Upper panels: a nutrient-like tracer $\tau$ with a photosynthesis-like sink term $(\nabla \tau$ is positive downward) in the upper ocean is subjected to a mesoscale (a) and submesoscale (b) vertical oscillation (respectively of amplitude $\delta z^{m}$ and $\delta z^{s}$ ). Lower panels: a tracer with no sink-source term is subjected to a mesoscale (c) and submesoscale (d) vertical oscillation, with a diabatic redistribution of tracer through shear driven mixing (purple arrows). In all panels, the horizontal direction represent time, increasing from left to tight. Positive (resp., negative and null) tracer concentration anomalies $(\delta \tau)$ relative to the parcel depth are represented with green (resp. orange and white) colors. + and - symbols also provide indications on tracer anomalies (resp. positive and negative). In limit case 2, submesoscale oscillation have a lesser impact than mesoscale ones while the opposite may be true in limit case 3 if submesoscale oscillations are more effective to produce shear-driven turbulence. 

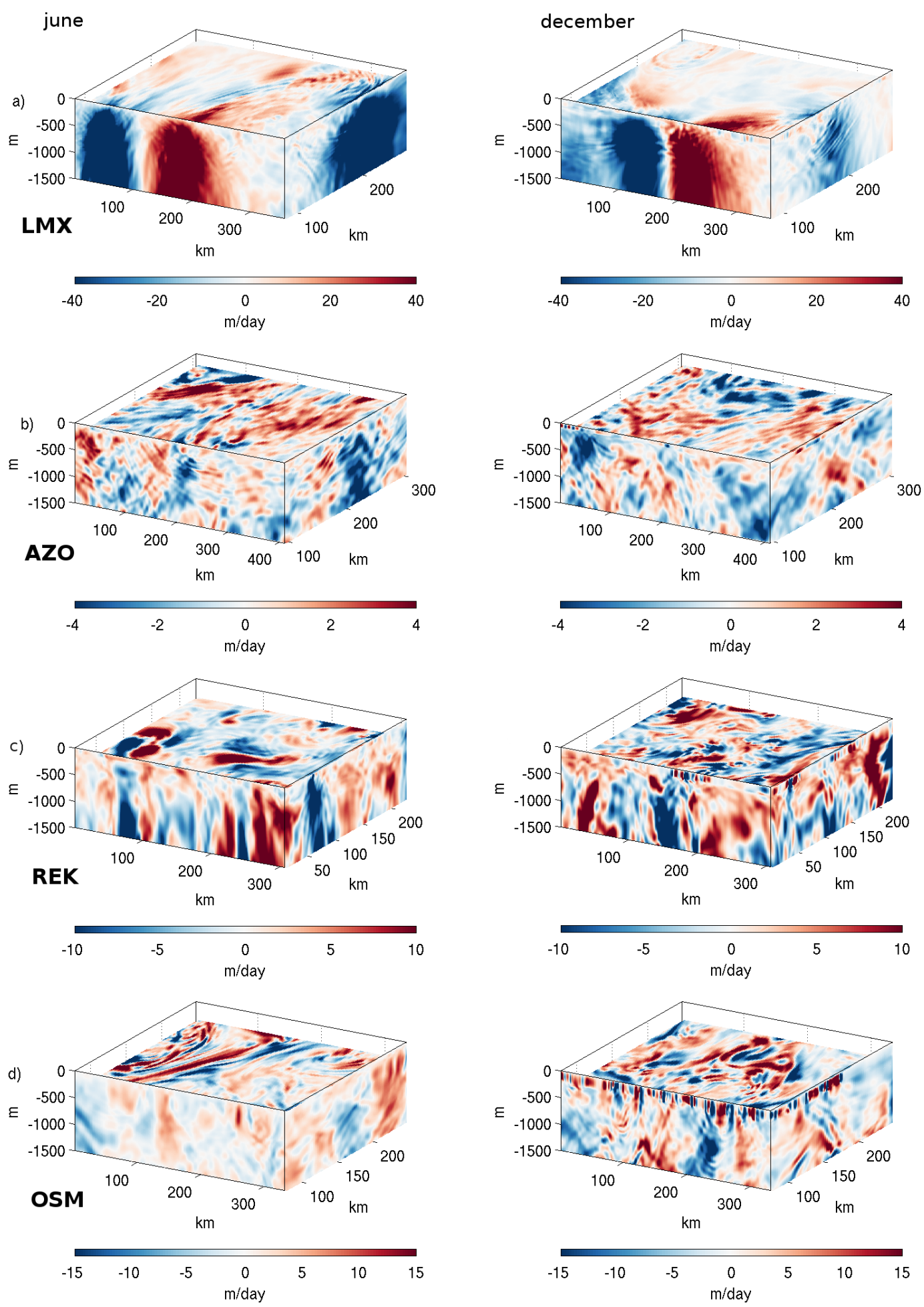

FIG. 1. Model vertical velocity on 10 june 2008 (left) and 10 december 2008 (right) in the a) LMX region, b) AZO region, c) REK region and d) OSM region. The upper horizontal plan corresponds to the depth of analysis $\left(z_{a}=220 \mathrm{~m}\right.$ in LMX, $250 \mathrm{~m}$ in AZO, and $380 \mathrm{~m}$ in REK and OSM) 


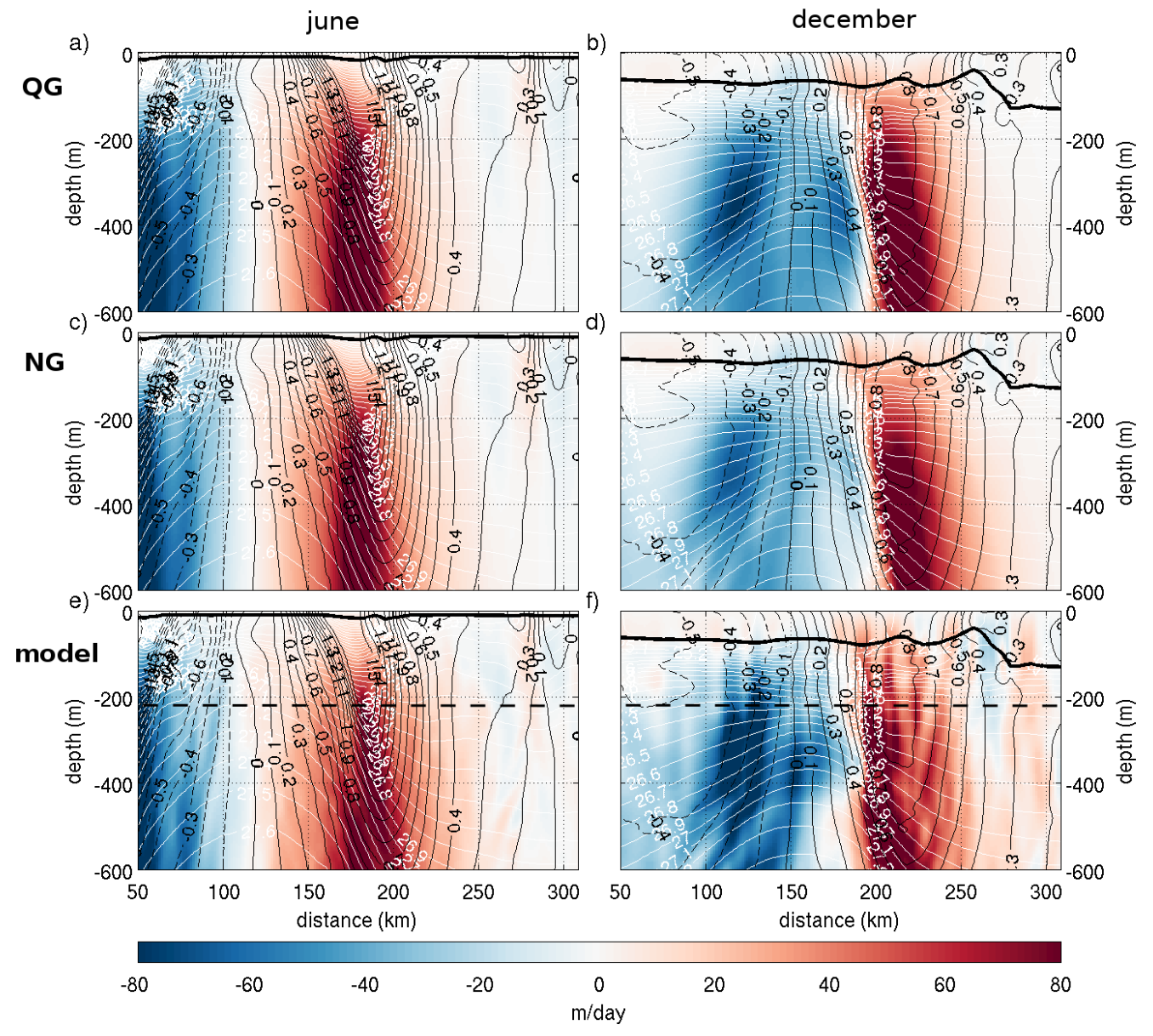

FIG. 2. Vertical velocity in the LMX region on 10 June 2008 (left, a, c, e) and 10 December 2008 (right, b, $\mathrm{d}, \mathrm{f})$ for the QG inversion (a, b), NG inversion (c, d) and model data (e, f). The thin white (resp. black) lines represent the isopycns (resp. horizontal velocity, $\mathrm{m} \mathrm{s}^{-1}$ ). The bold solid (resp. dashed) black line indicates 1208 mixed layer base (resp. the analysis depth $z_{a}$ ). 


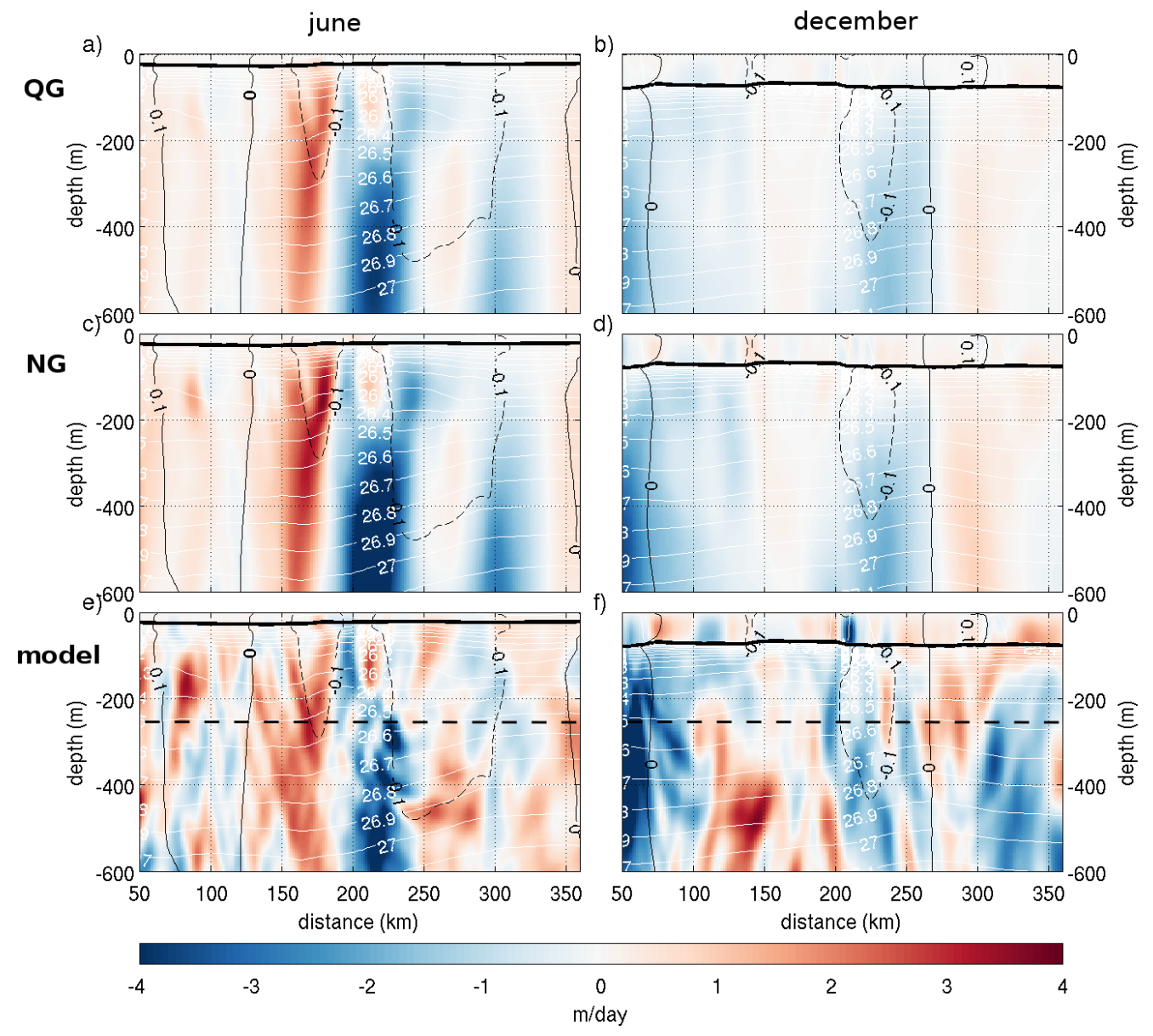

FIG. 3. As in Fig. 2 but for the AZO region. 


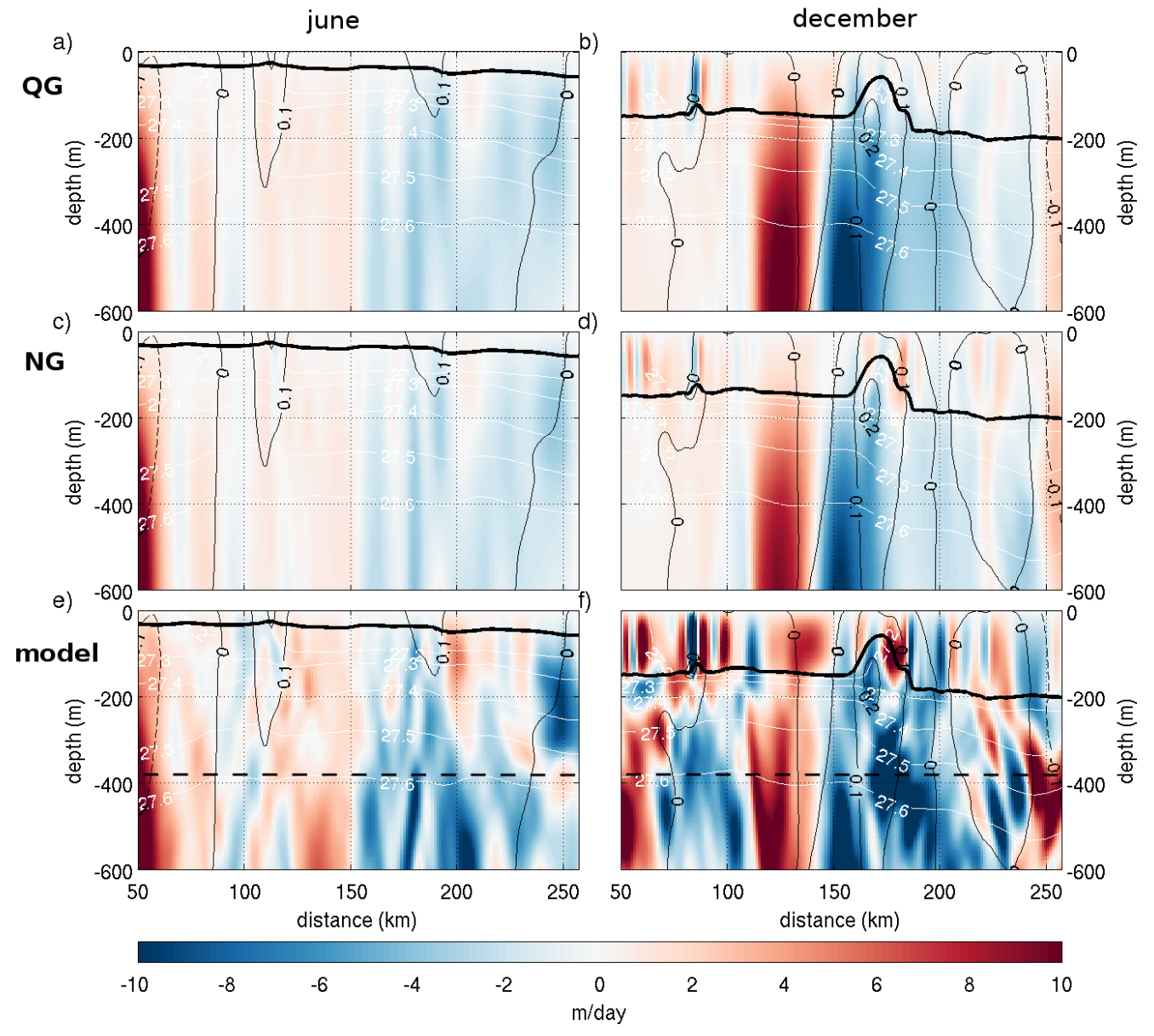

FIG. 4. As in Fig. 2 but for the REK region. 


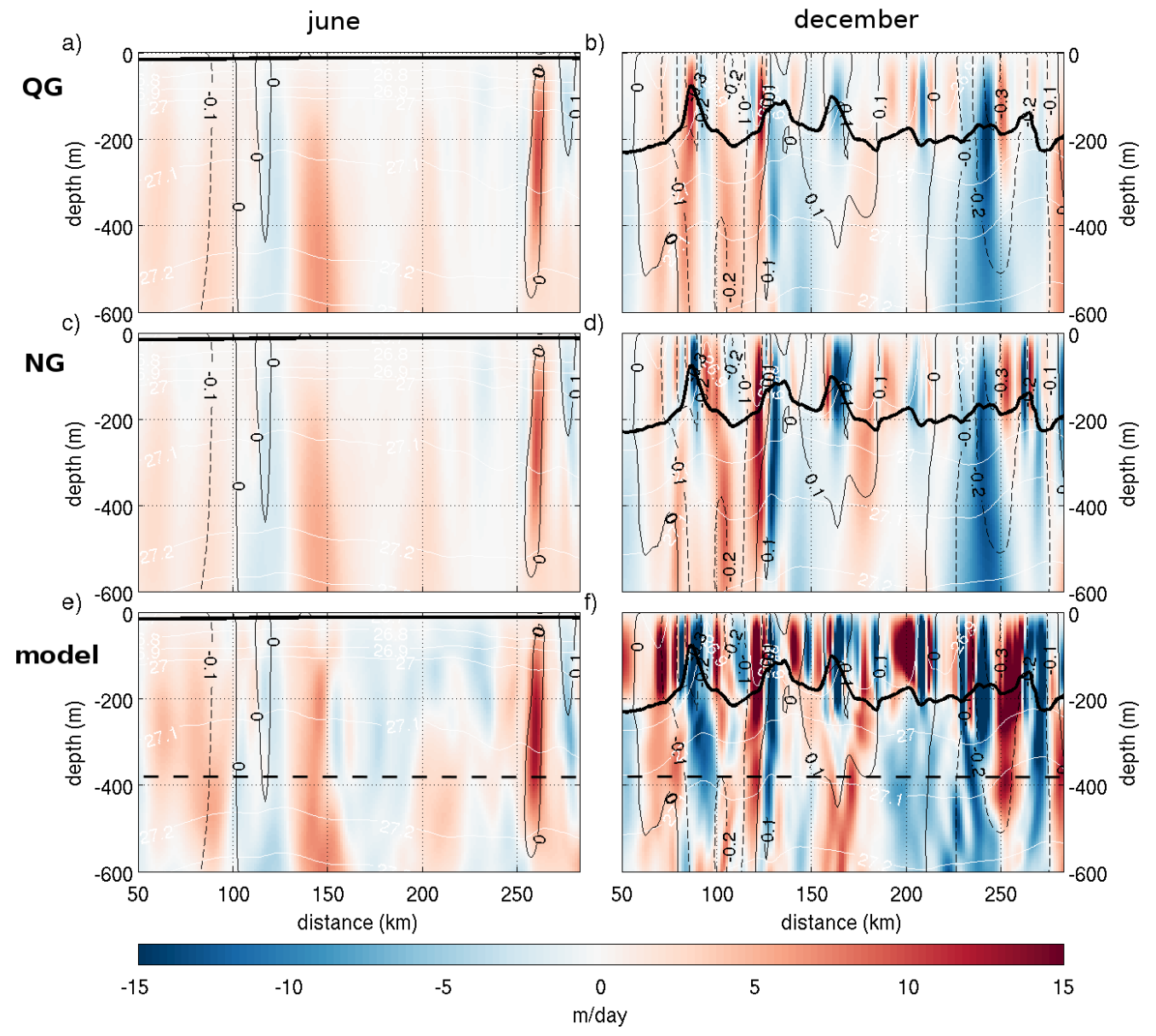

FIG. 5. As in Fig. 2 but for the OSM region. 

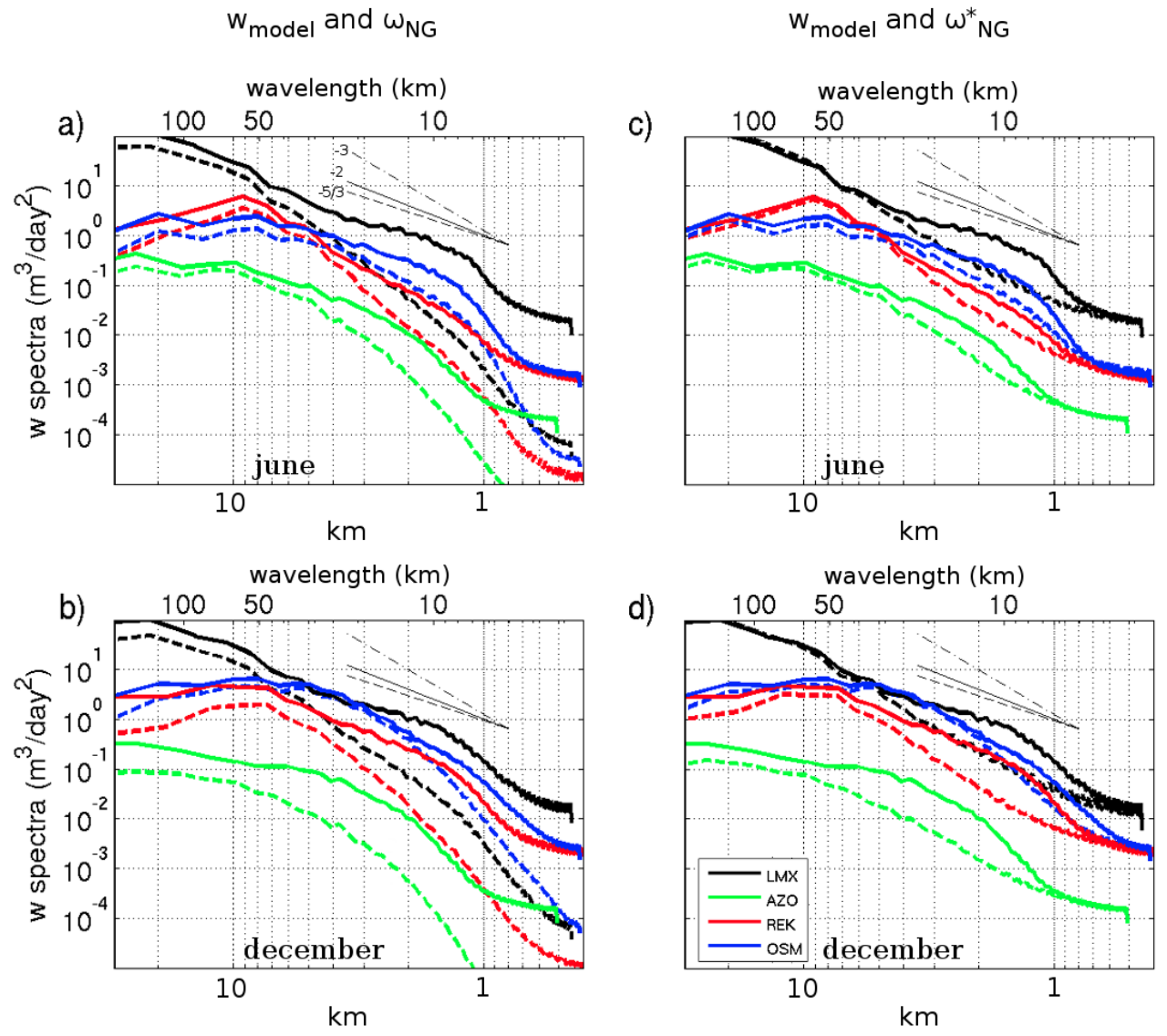

FIG. 6. a,b) Vertical velocity variance power spectra for $w_{\text {model }}$ (solid) and $\omega_{N G}$ (dashed) at depths $z_{a}$ in the

LMX (black, $z_{a}=220 \mathrm{~m}$ ), AZO (green, $z_{a}=250 \mathrm{~m}$ ), REK (red, $z_{a}=380 \mathrm{~m}$ ), and OSM (blue, $z_{a}=380 \mathrm{~m}$ ) regions in a) June and b) December. c,d) Same as $(\mathrm{a}, \mathrm{b})$ but for $w_{\text {model }}$ (solid) and $\omega_{N G}^{*}$ (dashed, the reconstructed $\omega_{N G}^{*}$ is computed using perfect boundary conditions). Straight (resp. dashed and dot-dashed) lines indicate -2 (resp. $-5 / 3$ and -3 ) roll-offs. The bottom (resp. top) horizontal axis displays the length scale (resp. wavelength). 

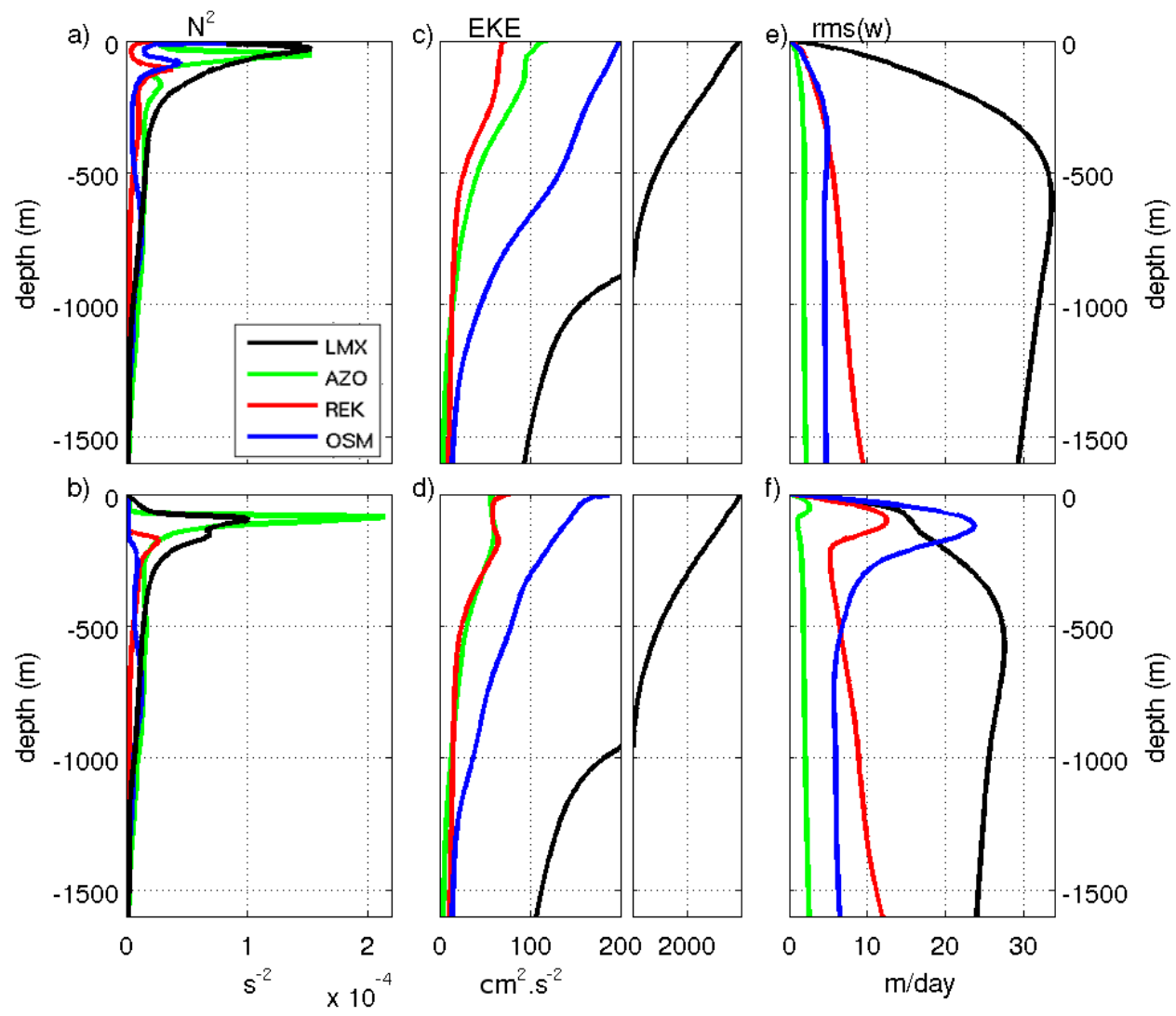

FIG. 7. Averaged vertical profiles of $(a, b)$ buoyancy frequency, $(c, d)$ eddy kinetic energy and (e,f) vertical velocity root mean square for the LMX (black), AZO (green), REK (red) and OSM (blue) regions in June (upper panel) and December (lower panel). Note the subpanels with a change of scale to accommodate the large differences in EKE between LMX and the other regions. 

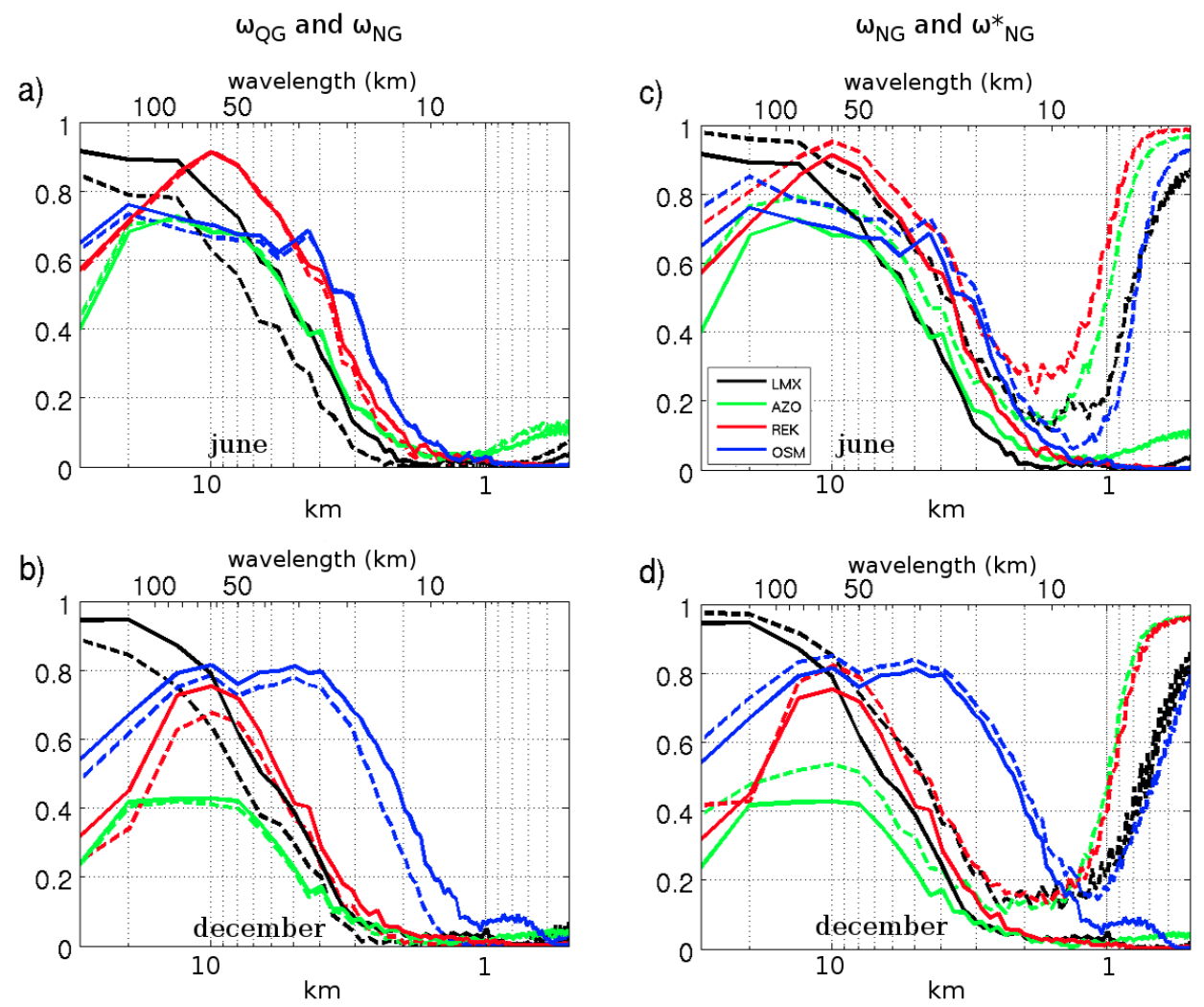

FIG. 8. a,b) Spectral coherence as a function of scale between $w_{\text {model }}$ and $\omega_{N G}$ (resp. $\omega_{Q G}$ ) at depths $z_{a}$ is represented by a solid (resp. dashed) line in the LMX (black), AZO (green), REK (red), and OSM (blue) regions in a) June and b) December. c,d) Same as (a,b) but the dahed line represent the coherence between $w_{\text {model }}$ and $\omega_{N G}^{*}$ (the reconstructed $\omega_{N G}^{*}$ is computed using perfect boundary conditions). The bottom (resp. top) horizontal axis displays the length scale (resp. wavelength). 

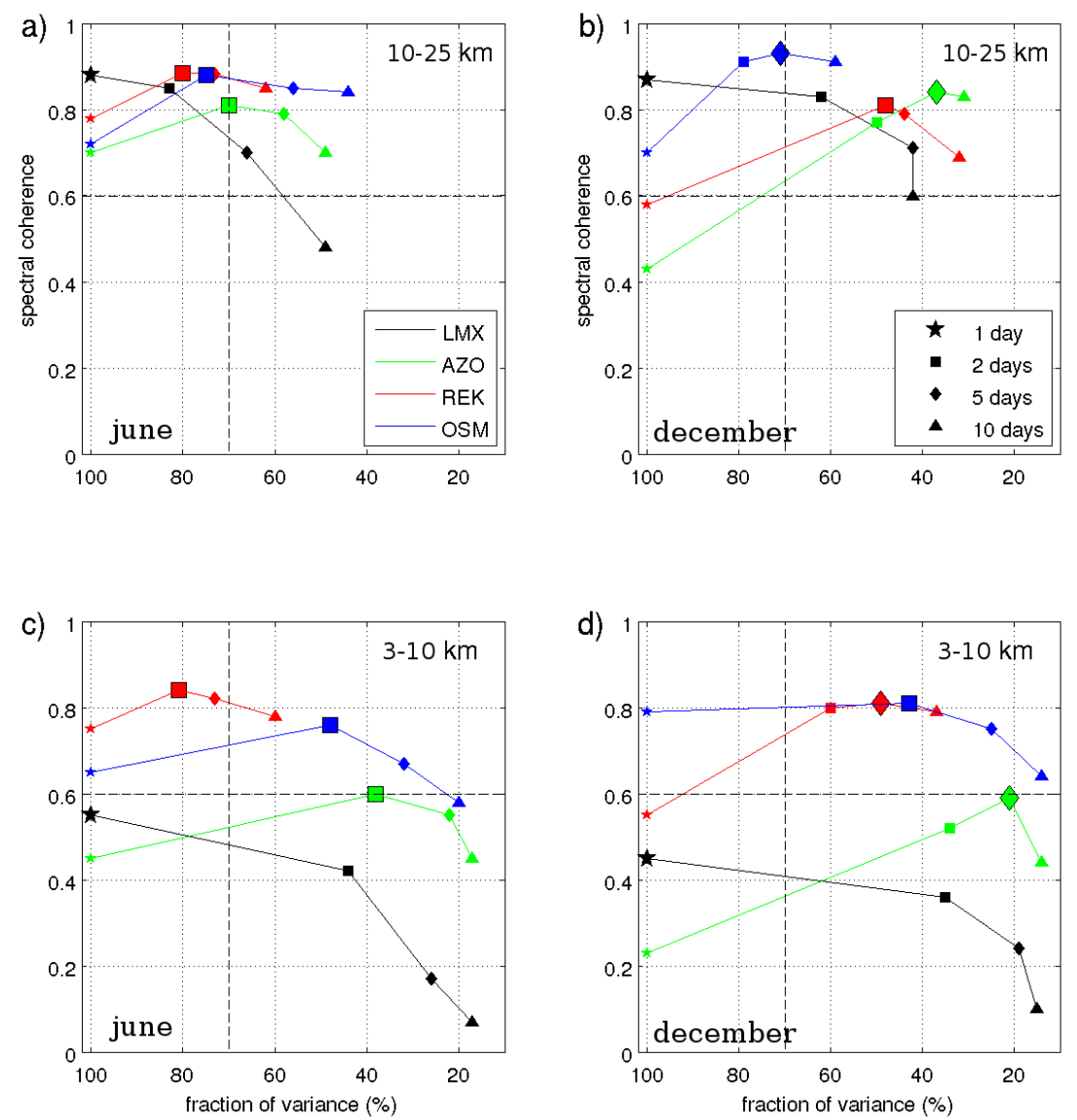

FIG. 9. Spectral coherence between $w_{\text {model }}$ and $\omega_{N G}$ at depths $z_{a}$ averaged over the scale ranges (a, b) $10-25$ shown for the LMX (black), AZO (green), REK (red), and OSM (blue) regions in June (left, a, c) and December (right, b, d). The markers represent the timespan of the averages: the star is 1 day, square 2 days, diamond 5 days and triangle 10 days. The highest coherence is indicated by a larger marker with a black contour. The black dashed lines mark a fraction of variance of $75 \%$ and a coherence of 0.6 . 

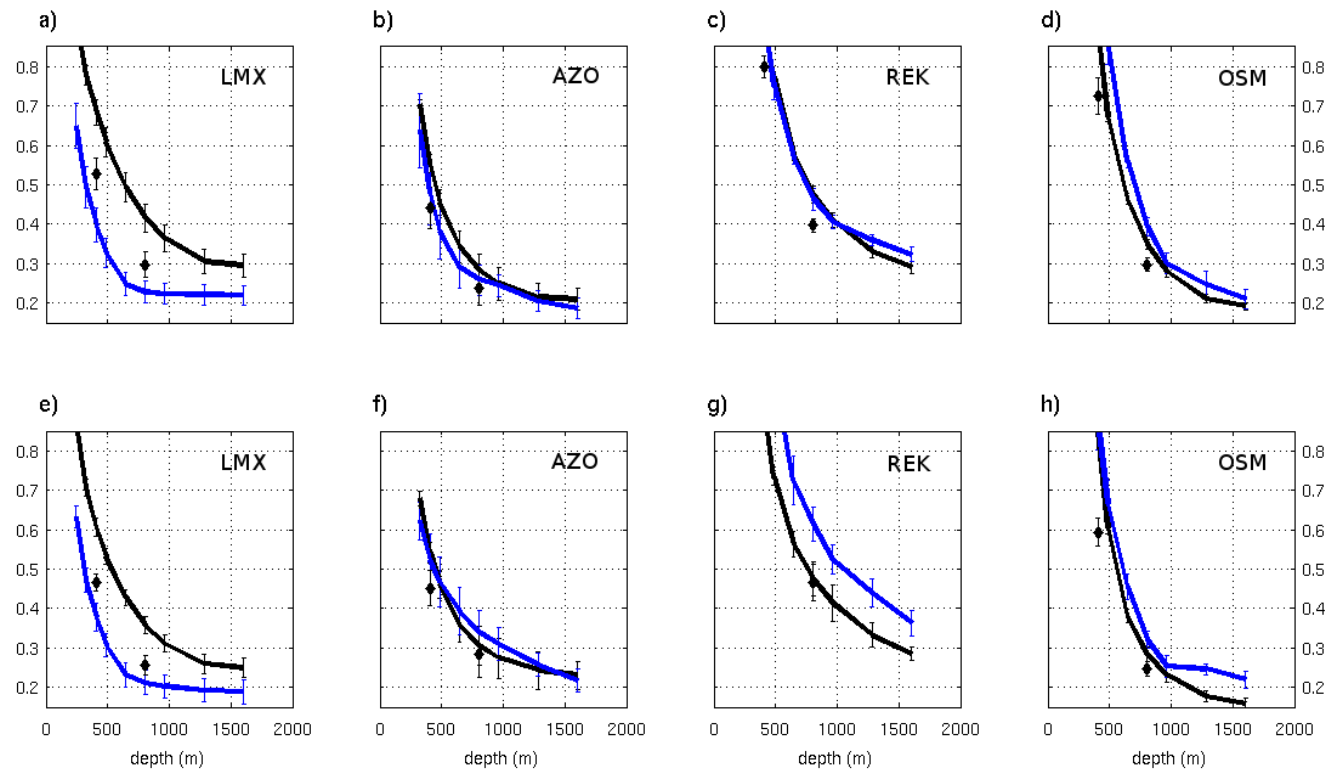

FIG. 10. Relative error, at depths $z_{a}$, between $w_{\text {model }}$ and $\omega_{\uparrow}$ in June (a-d) and December (e-h) in the LMX

$(\mathrm{a}, \mathrm{e}), \operatorname{AZO}(\mathrm{b}, \mathrm{f})$, REK $(\mathrm{c}, \mathrm{g})$ and $\operatorname{OSM}(\mathrm{d}, \mathrm{h})$ regions as a function of the depth where the bottom boundary condition $\left(z_{\text {bottom }}\right.$ in the text) is imposed. Errors for a Dirichlet (resp. Neumann) BBC are represented with a black (resp. blue) line. Black diamonds indicate error values for a Dirichlet boundary condition modified as in Rudnick (1996) (see section 4d for details). The vertical bars show the standard deviation over the 11 daily averages used for our analysis. 

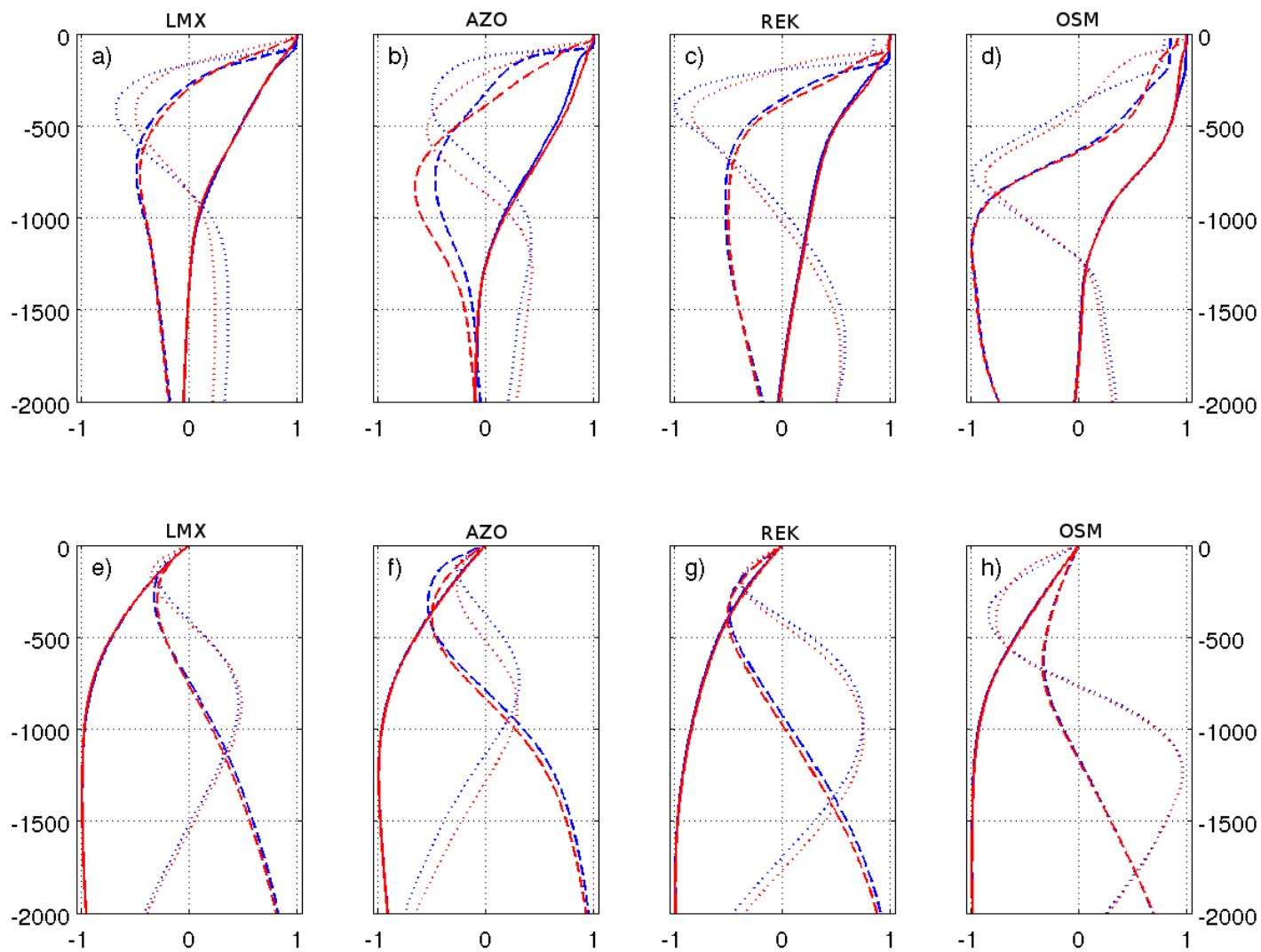

FIG. 11. First three baroclinic pressure modes for the a) LMX, b) AZO, c) REK, and d) OSM region in June (red) and December (blue). First three vertical velocity modes for the e) LMX, f) AZO, g) REK, and h) OSM region in June (red) and December (blue). The first mode (resp. 2nd and 3rd modes) is represented with a solid (resp. dashed and dotted) line. The amplitudes of the modes have been scaled so as to vary between -1 and 1 . 


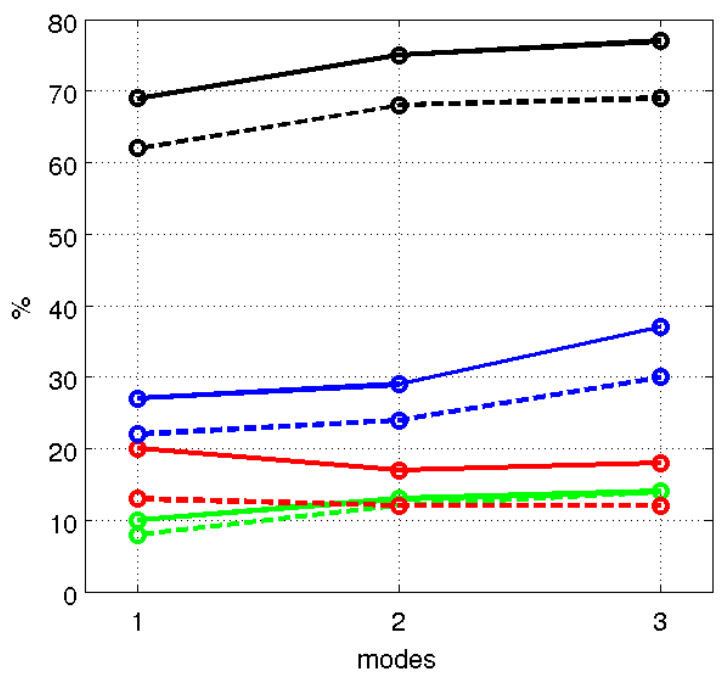

FIG. 12. Percentage of vertical water profiles for which the projection of model $w$ on the subset of the gravest vertical modes (1, 2 or 3 gravest modes) leads to an approximation of $w$ with a relative error that is less than 50 $\%$, i.e., percentage of profiles where $\varepsilon / w<0.5$ ( $\varepsilon$ being defined in equation A8). Percentages are represented for LMX (black), AZO (green), REK (red) and OSM (blue) in summer (plain lines) and winter (dashed lines). 


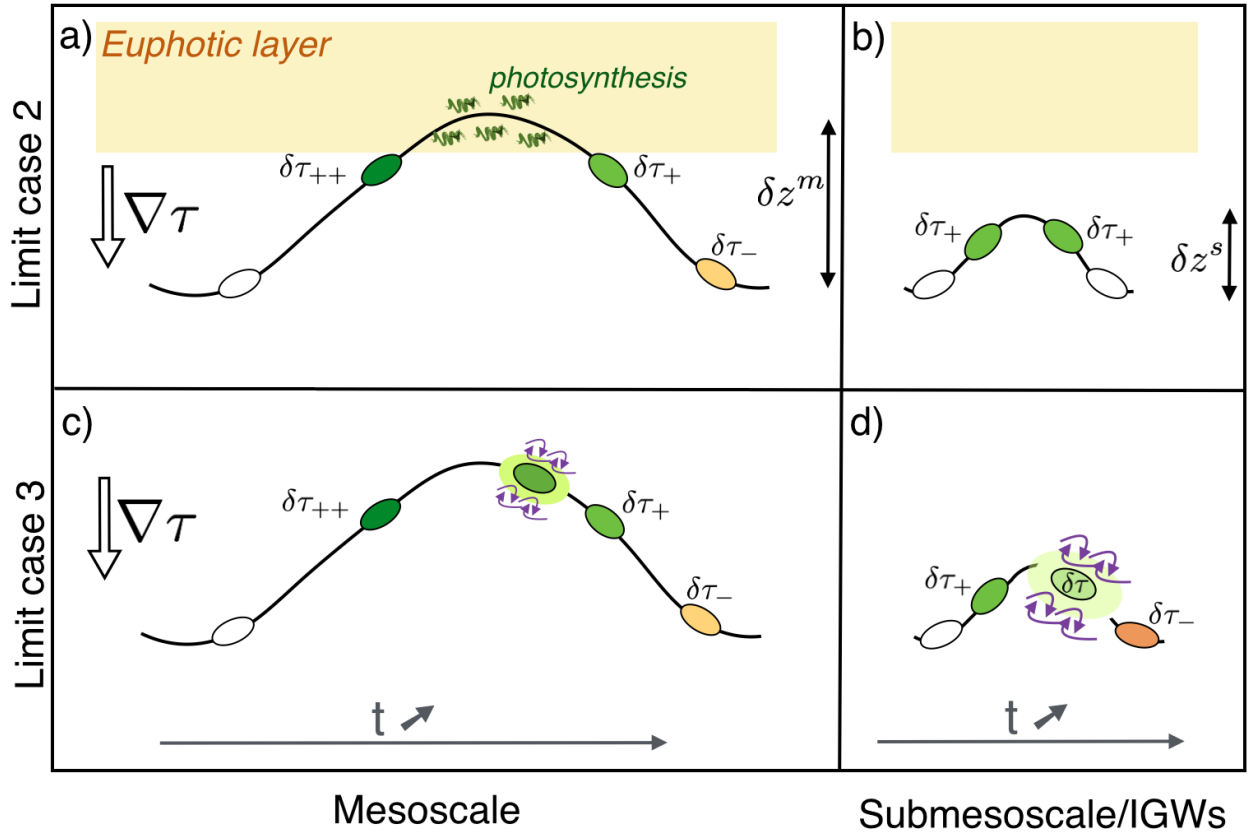

FIG. 13. schematic representation of the vertical disturbance (y-direction) and associated tracer anomaly temporal evolution undergone by a water parcel in 4 different situations. Upper panels: a nutrient-like tracer $\tau$ with a photosynthesis-like sink term ( $\nabla \tau$ is positive downward) in the upper ocean is subjected to a mesoscale (a) and submesoscale (b) vertical oscillation (respectively of amplitude $\delta z^{m}$ and $\delta z^{s}$ ). Lower panels: a tracer with no sink-source term is subjected to a mesoscale (c) and submesoscale (d) vertical oscillation, with a diabatic redistribution of tracer through shear driven mixing (purple arrows). In all panels, the horizontal direction represent time, increasing from left to tight. Positive (resp., negative and null) tracer concentration anomalies $(\delta \tau)$ relative to the parcel depth are represented with green (resp. orange and white) colors. + and - symbols also provide indications on tracer anomalies (resp. positive and negative). In limit case 2, submesoscale oscillation have a lesser impact than mesoscale ones while the opposite may be true in limit case 3 if submesoscale oscillations are more effective to produce shear-driven turbulence. 